\title{
Article \\ Molecular Genetic Approach and Evaluation of Cardiovascular Events in Patients with Clinical Familial Hypercholesterolemia Phenotype from Romania
}

\author{
Cristiana-Elena Vlad ${ }^{1,2,+}$, Liliana Georgeta Foia ${ }^{1,3, * \mathbb{C}}$, Roxana Popescu ${ }^{1,+}\left(\mathbb{D}\right.$, Ioana Popa ${ }^{1}$, Ruxandra Aanicai ${ }^{1}$, \\ Delia Reurean-Pintilei ${ }^{1}$, Vasilica Toma ${ }^{1}$, Laura Florea ${ }^{1,2}$, Mehmet Kanbay ${ }^{4}$ and Adrian Covic ${ }^{1,2}$ \\ 1 Faculty of Medicine, “Grigore T Popa" University of Medicine and Pharmacy, University Street, No 16, \\ 700115 Iasi, Romania; vladcristiana@gmail.com (C.-E.V.); roxana.popescu2014@gmail.com (R.P.); \\ ioanap93@yahoo.com (I.P.); ruxi03@yahoo.co.uk (R.A.); drdeliapintilei@gmail.com (D.R.-P.); \\ vasilicatoma40@yahoo.com (V.T.); lflorea68@yahoo.com (L.F.); accovic@gmail.com (A.C.) \\ 2 Department of Nephrology-Internal Medicine, “Dr. C.I. Parhon” Clinical Hospital, Carol I Street, No 50, \\ 700503 Iasi, Romania \\ 3 Department of Biochemistry, “Sf. Spiridon” Clinical County Hospital, Independentei Street, \\ 700111 Iasi, Romania \\ 4 Department of Medicine, Division of Nephrology, Koc University School of Medicine, 34450 Istanbul, Turkey; \\ mkanbay@ku.edu.tr \\ * Correspondence: lilifoia@yahoo.co.uk; Tel.: +40-744704452 \\ + These authors contributed equally to this work.
}

Citation: Vlad, C.-E.; Foia, L.G.; Popescu, R.; Popa, I.; Aanicai, R.; Reurean-Pintilei, D.; Toma, V.;

Florea, L.; Kanbay, M.; Covic, A. Molecular Genetic Approach and Evaluation of Cardiovascular Events in Patients with Clinical Familial Hypercholesterolemia Phenotype from Romania. J. Clin. Med. 2021, 10, 1399. https://doi.org/10.3390/ jcm10071399

Academic Editor: Karol Kaminski

Received: 4 March 2021

Accepted: 28 March 2021

Published: 31 March 2021

Publisher's Note: MDPI stays neutral with regard to jurisdictional claims in published maps and institutional affiliations.

Copyright: (c) 2021 by the authors. Licensee MDPI, Basel, Switzerland. This article is an open access article distributed under the terms and conditions of the Creative Commons Attribution (CC BY) license (https:// creativecommons.org/licenses/by/ $4.0 /)$.
Abstract: This study identifies the genetic background of familial hypercholesterolemia (FH) patients in Romania and evaluates the association between mutations and cardiovascular events. We performed a prospective observational study of 61 patients with a clinical diagnosis of FH selected based on Dutch Lipid Clinic Network (DLCN) and Simon Broome score between 2017 and 2020. Two techniques were used to identify mutations: multiplex ligation-dependent probe amplification (MLPA) and Sanger sequencing. The mutation rate was $37.7 \%$, i.e., 23 patients with mutations were identified, of which 7 subjects had pathogenic mutations and 16 had polymorphisms. Moreover, 10 variants of the low-density lipoprotein receptor ( $L D L R)$ gene were identified in 22 patients, i.e., one variant of the proprotein convertase subtilisin/kexin type 9 (PCSK9) gene in six patients, and one variant of the apolipoprotein $\mathrm{B}(A P O B)$ gene in three patients. Of the $L D L R$ gene variants, four were $L D L R$ pathogenic mutations (c.81C > G, c.502G > A, c.1618G > A mutations in exon 2, exon 4, exon 11, and exon 13-15 duplication). The PCSK9 and $A P O B$ gene variants were benign mutations. The pathogenic $L D L R$ mutations were significant predictors of the new cardiovascular events, and the time interval for new cardiovascular events occurrence was significantly decreased, compared to FH patients without mutations. In total, 12 variants were identified, with four pathogenic variants identified in the LDLR gene, whereas $62.3 \%$ of the study population displayed no pathological mutations.

Keywords: familial hypercholesterolemia; cardiovascular events; pathogenic mutations; molecular genetic

\section{Introduction}

Familial hypercholesterolemia (FH) (Online Mendelian Inheritance in Man-OMIM 143890) is an autosomal dominant genetic pathology, frequently caused by pathogenic variants of the low-density lipoprotein receptor (LDLR), apolipoprotein B (APOB), and the proprotein convertase subtilisin/kexin type 9 (PCSK9) [1-9]. In Europe, the FH frequency with the heterozygous form varies from 1:200 to 1:500 individuals, being extremely rare in the homozygous form, which ranges from 1:300,000 to 1:1.000,000 in the general population $[4,5,7,10]$. These pathological variants trigger elevated low-density lipoprotein cholesterol (LDL-C) levels, leading to accelerated atherosclerosis and atherosclerotic cardiovascular disease (ASCVD) [1,5-8,11]. Atherosclerosis is a complex multifactorial disorder 
consisting of the chronic inflammatory response, which then causes plaque formation in the intima and media of medium and large arteries [12]. Hypercholesterolemia causes the proliferation of hematopoietic and progenitor cells, leading to leukocytosis and increased atherosclerosis [13]. Thereby, patients with high LDL-C values and low high-sensitivity C-reactive protein (hsCRP) levels have a lower risk of stroke, coronary heart disease (CHD), and death from CHD, compared to those with high levels of LDL-C and hsCRP [13]. The clinical and biological diagnosis of FH is based on the Dutch Lipid Clinic Network (DLCN) score, Simon Broome, and US MedPed [6].

The low-density lipoprotein receptor (LDLR) is a cell surface glycoprotein that mediates the specific binding and uptake of apoB-100 lipoproteins by receptor-mediated endocytosis [14]. The LDLR gene locus is located on chromosome 19p13.1-13.3, with 18 exons and 17 introns, encoding a mature protein of 839 amino acids and including a signal sequence of 21 amino acids with a series of five discrete major structural domains $[6-8,10,14]$. LDLR gene mutations are the most common $\mathrm{FH}$ cause (90-95\%), whereas $A P O B$ mutations account for only 3-6\%, and mutations in PCSK9 are found in less than $1-3 \%$ of patients $[2,15]$. The diversity of the underlying variants is wide; over 2000 variants have been documented for $L D L R$, with monogenic and polygenic forms and increased FH penetrance, yet the expressivity varies depending on the nature of the mutation $[3,6,7,15]$. Each country has its specific spectrum of $L D L R$ mutations [3]. In addition, the mutations located in the $A P O B$ gene alter the functional activity of the apolipoprotein $\mathrm{B}$, decreasing its binding to LDLR and reducing the clearance of LDL particles and the accumulation of LDL-C [16]. PCSK9 is a serine protease of the subtilase family, secreted primarily by the liver and kidney via sterol regulatory element-binding protein 2 (SREBP-2) modulation and, by a second transcription factor, the hepatocyte nuclear factor 1 (HNF1) [17-19]. The PCSK9 plasma levels are closely related to nutritional and hormonal status (e.g., hepatic glucagon receptor signaling) and diurnal rhythm [19]. The azacytidine activation associated with deacetylase sirtuin 1 contributes to reduced PCSK9 secretion, confirming the important role of epigenetic regulation by DNA methylation and histone acetylation [19]. PCSK9 is a novel therapeutic target for familial hypercholesterolemia designed for lowering cardiovascular risk (PCSK9 is observed in carotid atherosclerotic lesions) through monoclonal antibodies and small interfering RNA $[19,20]$. PCSK9 determines the degradation of LDLR and inhibits receptor recycling in the hepatocyte membrane [19-21].

The early diagnosis and initiation of adequate lipid-lowering drugs are based on a proper understanding of the molecular mechanisms of this disease and contribute to a significant reduction in cardiovascular morbidity and mortality $[4,8]$. The cascade screening system has been used in many European countries (e.g., the Netherlands, Norway, Iceland, Switzerland, the United Kingdom, and Spain) as an effective way to identify FH patients [4,6]. However, in some European countries (e.g., Romania), FH is still underdiagnosed and mistreated [4,6]. In Romania, the genetic characterization of FH has not been explored adequately because there are no studies on the molecular aspects of this pathology. Our prospective observational study included patients with $\mathrm{FH}$, with the following objectives: (a) to identify the mutation in order to establish the genetic background of FH patients in Romania and (b) to evaluate the association between the identified mutations and the cardiovascular events.

\section{Materials and Methods}

\subsection{Patient Recruitment}

The study was designed as an observational, prospective, three-year study (October 2017 to October 2020) in three referral centers from the northeastern region of Romania, which includes eight counties and a population of over 3,980,000 inhabitants.

The study population included 980 patients with dyslipidemia who were identified between September 2016 and October 2017, and 61 patients meeting the following inclusion criteria: (a) subjects with full mental capacity who signed the informed consent form and (b) men and women aged over 18 years. The DLCN score above 3 and the Simon Broome 
criteria (probable or possible FH) represented two important selection tools for the patients with a clinical diagnosis of $\mathrm{FH}$. These criteria included the following elements: identification of a family history of hypercholesterolemia or cholesterol deposits in vascular and extravascular tissues; setup of a personal history of early onset of coronary, cerebrovascular, and peripheral vascular diseases; clinical observations regarding the presence of either xanthomas, xanthelasma and/or arcus cornealis; biological identification of total cholesterol (TC) $>300 \mathrm{mg} / \mathrm{dL}, \mathrm{LDL}-\mathrm{C}>190 \mathrm{mg} / \mathrm{dL}$ without treatment, or $>100 \mathrm{mg} / \mathrm{dL}$ following treatment with maximum doses of statins ( $40 \mathrm{mg}$ rosuvastatin, $80 \mathrm{mg}$ atorvastatin), in combination with ezetimibe.

Exclusion criteria included the following: subjects lacking discernment or those who refused to sign the informed consent; patients under the age of 18; pregnant and breastfeeding women; subjects with severe physical disabilities, dementia, neoplasms, and other causes of secondary hypercholesterolemia (uncontrolled diabetes, nephrotic syndrome, hypothyroidism, drug-induced dyslipidemia) [22].

\subsection{Clinical and Biological Evaluation of FH Patients}

The patients included in the study were coded with the letter $\mathrm{H}$ and the corresponding ID number. The medical history revealed that certain patients had received antihypertensive medication (those with $\mathrm{BP}>140 / 90 \mathrm{mmHg}$ ) or oral antidiabetic medication (those diagnosed with type 2 diabetes), which was allowed throughout the study according to the specialist doctors' prescription.

The study included patients with a DLCN score $>3$ for the FH population $[23,24]$. The reference values of the DLCN score were as follows: $3-5$ points highlighted possible $\mathrm{FH}$, 6-7 points indicated probable $\mathrm{FH}$, while over 8 points indicated definite $\mathrm{FH}[23,24]$. The other score, namely, the Simon Broome score, flagged the presence of possible, probable, or definitive $\mathrm{FH}[23,24]$.

Laboratory tests included values at baseline, and at 12, 24, and 36 months; total cholesterol mg/dL, LDL-C mg/dL, high density cholesterol lipoprotein (HDL-C) mg/dL, triglycerides (TG) $\mathrm{mg} / \mathrm{dL}$, blood glucose $(\mathrm{mg} / \mathrm{dL}$ ), and high-sensitivity C-reactive protein (hsCRP) $\mathrm{mg} / \mathrm{dL}$ were measured by spectrophotometric assay (Architect c8000-Abbott Laboratory, Chicago, IL, USA).

\subsection{Evaluation of the New Cardiovascular Events}

Atherosclerotic cardiovascular disease (ASCVD) was defined as a history of one of the following diseases, as identified in the medical records: coronary heart disease (CHD) (with particularities such as acute coronary syndrome, myocardial infarction (MI), stable angina, coronary revascularization, ischemic stroke, or transient ischemic attack) and peripheral artery disease (PAD) [25].

Further explorations for cardiovascular evaluation included the following:

- electrocardiogram (ECG) for ischemic changes assessment;

- ankle-brachial index (ABI) measurement with a sphygmomanometer and a portable ultrasonography device for determining sounds that detect systolic blood pressure in the lower limbs; the reference ABI values were between 0.9 and 1.3;

- echocardiography (Siemens Acuson CV70 Cardiac Vascular Ultrasound Machine), highlighting left ventricular (LV) wall motion abnormalities and ejection fraction values, important predictors of left ventricular systolic dysfunction;

- measurement of carotid intima-media thickness (cIMT) (at the levels of the carotid bifurcation, internal, external, right and left carotid arteries) by using Siemens Acuson CV70 Cardiac Vascular Ultrasound Machine, B-mode and color Doppler ultrasound $(5-10 \mathrm{MHz})$. The average of the cIMT (the average of the six quantified segments) was also recorded. The reference cIMT values were under $0.9 \mathrm{~mm}[26,27]$. 


\subsection{Evaluation of the Mutations in the LDLR, APOB, and PCSK9 Genes}

\subsubsection{DNA Genomic Extraction}

DNA was extracted from $3 \mathrm{~mL}$ of peripheral blood samples stored with EDTA agent, using Wizard Genomic DNA Purification Kit (Promega Corp., Madison, WI, USA).

\subsubsection{MLPA (P062, LDLR MLPA Kit, MRC Holland, Amsterdam, Netherlands)}

The probe mix P062 LDLR was used for the deletion of or duplication in the LDLR genes. The multiplex ligation-dependent probe amplification (MLPA) analysis was performed according to the manufacturer's protocol. The genomic DNA was denatured and hybridized with P062 probes at $60^{\circ} \mathrm{C}$ for approximately $17 \mathrm{~h}$. The PCR amplification was performed after $15 \mathrm{~min}$ ligation at $54^{\circ} \mathrm{C}$, using Cy5 labeled primers. Fluorescent amplification products were separated based on their length by capillary electrophoresis in a CEQ 8000 GeXP Genetic Analysis System (Beckman Coulter, Brea, CA, USA), and the results were analyzed using the Coffalyser.NET program (MRC-Holland, Amsterdam, The Netherlands). The probe ratios of deletion and duplication were fixed at 0.7 and 1.3, respectively [28].

\subsubsection{Sanger Sequencing}

The LDLR gene coding region and intron-exon boundaries were sequenced bi-directionally for all the patients. From the coding region were evaluated the exon 7 for PCSK9 and exon 26 for APOB.

Approximately $125 \mathrm{ng}$ genomic DNA was amplified in a $25 \mu \mathrm{L}$ reaction volume, on Sensoquest Thermocycler (Sensoquest, Göttingen, Germany), using GoTaq ${ }^{\circledR}$ G2 Hot Start Master Mix (Promega, Madison, WI, USA). The PCR conditions were initial denaturation $\left(10 \mathrm{~min}\right.$ at $\left.95^{\circ} \mathrm{C}\right)$, followed by 35 cycles of denaturation $\left(30 \mathrm{~s}\right.$ at $\left.94{ }^{\circ} \mathrm{C}\right)$, annealing varying between 55 and 66 degrees depending on the amplified fragments and elongation $(60 \mathrm{~s}$ at $72{ }^{\circ} \mathrm{C}$ ), with a final elongation at $72{ }^{\circ} \mathrm{C}$ for $5 \mathrm{~min}$. The sequencing was performed using GenomeLab DTCS-Quick Start Kit (Beckman Coulter, Brea, CA, USA) in a $10 \mu \mathrm{L}$ reaction volume. The PCR and sequencing products were purified with the Agencourt system (Beckman-Coulter, USA), Agencourt AMPure XP, and Agencourt Cleanseq ${ }^{\circledR}$ system, respectively. The final products were subsequently separated by capillary electrophoresis on CEQ 8000 GeXP Genetic Analysis System (Beckman-Coulter) [28]. Sequences were analyzed using MegaX software and were compared with the corresponding reference sequences, namely, NM_000527.5 for LDLR, NM_000384.3 for APOB, and NM_174936.3 for PCSK9. The variants were verified in Mutation taster, ClinVar, and PolyPhen for predicting the functional effect of DNA sequence alterations [28,29].

\subsection{Statistical Analysis}

The data of the FH patients were introduced into a database and processed by means of the statistical functions of the SPSS version 20.0 system. One-sample KolmogorovSmirnov for normal distribution tests were performed, with the data being calculated as mean and standard deviation (SD) for normal distribution variables, percent for categorical variables by using a frequency test, and median and interquartile range (IQR) for continuous variables with asymmetrical distribution. Bivariate correlation analysis was performed between the scale variables, using the Spearman correlation coefficient. To evaluate the associations between nominal variables, specific association coefficients were used (Cramer's, Phi, contingency coefficient, chi square $\left(\chi^{2}\right)$ ). Comparative analyses between the pathological history, clinical, and paraclinical history according to mutations were achieved for the values that did not meet the criteria of normal homogeneity. The normal distribution was performed for nonparametric tests, i.e., Mann-Withney U sample, Wilcoxon signed rank, Kruskall-Wallis H test, and Friedman test. Survival free of ASCVD, during follow up and according to mutations, was estimated using the Kaplan-Meier method. The duration of the follow up was calculated from the date of inclusion in the study to the date of the occurrence of cardiovascular events. Multiple logistic regression 
analysis was applied to detect the independent factors for cardiovascular events. The $p$-value $<0.05$ was considered statistically significant.

\section{Results}

\subsection{The Genetic Spectrum of FH in Romania}

The study group included 61 patients $(6.2 \%$ of all patients examined), with a mean age of $48.5 \pm 12.5$ years old, all subjects being Caucasian, with a higher proportion of women compared to men (63.9\% versus $36 \%$ ). Moreover, $36.1 \%$ of the patients had ASCVD history (Table 1). The laboratory results recorded TC $315 \pm 56 \mathrm{mg} / \mathrm{dL} ; \mathrm{LDL}-\mathrm{C} 254.2 \pm 53 \mathrm{mg} / \mathrm{dL}$; HDL-C $45.8 \pm 18 \mathrm{mg} / \mathrm{dL}$; and TG $174.4 \pm 92 \mathrm{mg} / \mathrm{dL}$ (for all patients), whereas the lipid profile (LDL-C, HDL-C, TG) did not differ according to mutations (Table 1). Furthermore, the $\mathrm{FH}$ patients who had pathogen/likely pathogen mutations had significantly increased TC levels and high DLCN score values, compared to mutation-free patients with a clinical diagnosis of $\mathrm{FH}$ and $\mathrm{FH}$ patients with benign/likely benign mutations (Table 1). Moreover, FH patients with pathogenic mutations had significantly increased TC levels and high values of DLCN score, compared to patients without mutations. The number of patients with pathogenic mutations, male, active smokers, with high blood pressure, cardiovascular history, obesity, and diabetes was reduced, compared to the number of subjects without mutations (Table 1). The same results were observed in patients with benign variants, compared to subjects without mutations. At baseline, all the patients had lipid-lowering therapies (about one year of treatment prior to inclusion in the study), the most frequent being the statin monotherapy (36.1\%), followed by associations between statin and ezetimibe, statin and fenofibrate, and their triple combination (Table 1). There were no significantly different percentages between patients with benign/pathogenic mutations versus patients without mutations who received lipid-lowering treatments.

In the study group with $61 \mathrm{FH}$ patients, mutations in $L D L R, A P O B, P C S K 9$ genes were analyzed by MLPA and Sanger sequencing, revealing pathological results in 23 cases, with a mutation detection rate of $37.7 \%$. Among these subjects, 16 patients had benign/pathogenic variants in LDLR gene, 4 patients in LDLR and PCSK9 genes, 1 subject in LDLR and APOB genes, 1 subject in $A P O B$ and PCSK9 genes, and 1 patient in all candidate genes (LDLR, $A P O B, P C S K 9)$ (Table 2 and Table S1). Regarding the clinical significance of FH-associated mutations in ClinVar, respectively, Leiden Open Source Variation Database (LVOD), out of the 23 patients identified with mutations, seven subjects had pathogenic/likely pathogenic mutations and the others had either benign/likely benign mutations or conflicting interpretations (Table 2 and Table S1). The pathogenic/likely pathogenic $L D L R$ mutations were detected in three patients having the same mutation in LDLR exon 11 (c.1618G > A homozygous) (Figure 1, Table 2 and Table S1), in two patients bearing the same mutation in LDLR exon 2 (c.81C > G heterozygous) (Figure 2, Table 2 and Table S1), one patient displaying mutation in LDLR exon 4 (c.502G > A heterozygous) (Figure 3, Table 2 and Table S1), and one patient showing duplication in exon 13-15 (c.(1845 + 1_1846-1)_(2311+1_2312-1)dup)) (Figure 4; Table 2 and Table S1). In this study, the c.1026A > G in the PCSK9 gene and the c.10740C > T in the APOB gene were benign/likely benign variants (Table 2 and Table S1). 


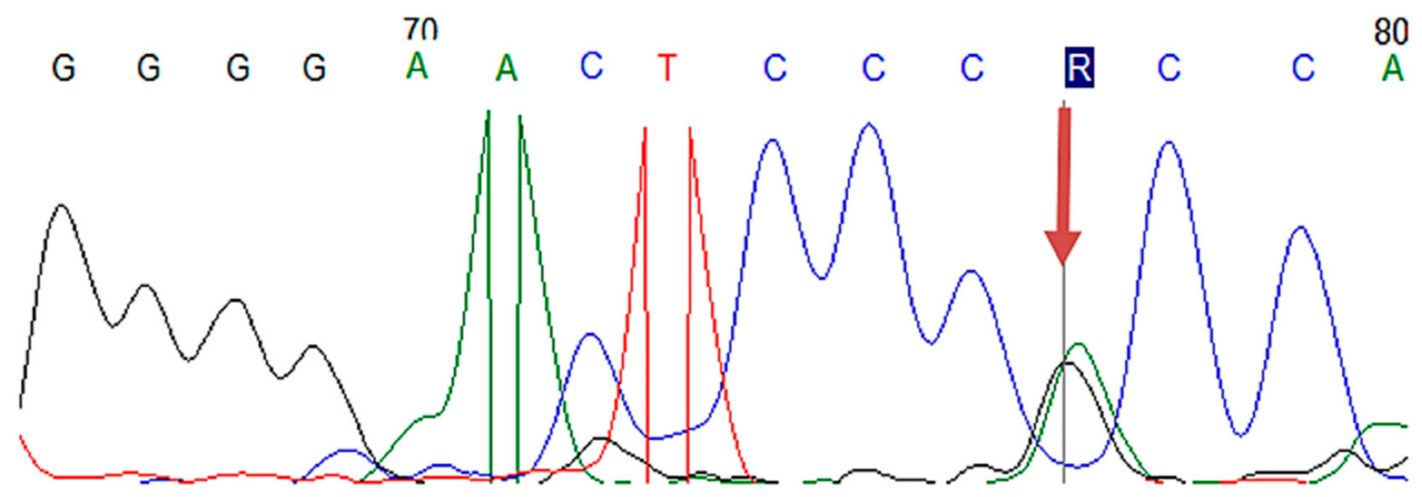

Figure 1. The Sanger sequencing electropherogram in patients H19, H46, and H53: pathogen mutation of LDLR in exon 11 (c.1618G > A).

Table 1. Baseline characteristics of familial hypercholesterolemia (FH) patients.

\begin{tabular}{|c|c|c|c|c|c|}
\hline \multirow[b]{2}{*}{ Characteristics } & \multicolumn{4}{|c|}{ Patients with FH } & \multirow[b]{2}{*}{$p$} \\
\hline & Overall & $\begin{array}{l}\text { Without } \\
\text { Mutation }\end{array}$ & $\begin{array}{c}\text { Benign/Likely } \\
\text { Benign } \\
\text { Mutation }\end{array}$ & $\begin{array}{c}\text { Pathogenic/Likely } \\
\text { Pathogenic } \\
\text { Mutation }\end{array}$ & \\
\hline$n$ & 61 & 38 & 16 & 7 & \\
\hline Age-yo (mean \pm SD) & $48.4 \pm 12.5$ & $50.3 \pm 11.6$ & $43.4 \pm 13.6$ & $50.1 \pm 13.4$ & 0.18 \\
\hline Gender (male) $n(\%)$ & $22(36.1 \%)$ & $14(36.8 \%)$ & $5(31.3 \%)$ & $3(42.9 \%)$ & 0.86 \\
\hline Smoker $n(\%)$ & $18(29.5 \%)$ & $12(31.6 \%)$ & $5(31.3 \%)$ & $1(14.3 \%)$ & 0.64 \\
\hline High blood pressure $n(\%)$ & $31(50.8 \%)$ & $22(57.9 \%)$ & $6(37.5 \%)$ & $3(42.9 \%)$ & 0.35 \\
\hline CHD history $n(\%)$ & $13(21.3 \%)$ & $10(26.3 \%)$ & $3(18.8 \%)$ & 0 & \multirow{3}{*}{0.06} \\
\hline PAD history $n(\%)$ & $9(14.8 \%)$ & $7(18.4 \%)$ & $2(12.5 \%)$ & 0 & \\
\hline CHD + PAD history $n(\%)$ & $14(23 \%)$ & $6(15.8 \%)$ & $3(18.8 \%)$ & $5(71.4 \%)$ & \\
\hline Obesity $n(\%)$ & $22(36.1 \%)$ & $14(36.8 \%)$ & $4(25 \%)$ & $4(57.4 \%)$ & 0.33 \\
\hline Type 2 diabetes $n(\%)$ & $8(13.1 \%)$ & $5(13.2 \%)$ & $2(12.5 \%)$ & $1(14.3 \%)$ & 0.99 \\
\hline Physical inactivity $n(\%)$ & $30(49.2 \%)$ & $21(55.3 \%)$ & $5(31.3 \%)$ & $4(57.1 \%)$ & 0.25 \\
\hline TC mg/dL (median \pm IQR) & $315 \pm 56$ & $307.5 \pm 44$ & $320 \pm 41$ & $353 \pm 206$ & $0.02 *$ \\
\hline $\mathrm{LDL}-\mathrm{C}$ mg/dL (mean $\pm \mathrm{SD})$ & $254.2 \pm 53$ & $246.2 \pm 46.2$ & $255.4 \pm 46.5$ & $294.7 \pm 85.1$ & 0.31 \\
\hline HDL-C mg/dL (median \pm IQR) & $45.8 \pm 18$ & $45 \pm 12.3$ & $48.5 \pm 16.1$ & $39 \pm 16.2$ & 0.28 \\
\hline $\mathrm{TG}$ mg/dL (mean $\pm \mathrm{SD})$ & $174.4 \pm 92$ & $179.5 \pm 92.2$ & $160.6 \pm 102.3$ & $178.9 \pm 74.2$ & 0.61 \\
\hline hsCRP mg/L (mean \pm SD) & $5.85 \pm 2.29$ & $5.8 \pm 2.2$ & $6.3 \pm 2.2$ & $7.4 \pm 2.4$ & 0.26 \\
\hline ECG changes $n(\%)$ & $25(41 \%)$ & $13(34.2 \%)$ & $7(43.8 \%)$ & $5(71.4 \%)$ & 0.18 \\
\hline $\mathrm{EF} \%($ mean $\pm \mathrm{SD})$ & $53.2 \pm 9.8$ & $53.8 \pm 9.4$ & $54.1 \pm 9.6$ & $47.6 \pm 11.8$ & 0.35 \\
\hline $\mathrm{ABI}($ mean $\pm \mathrm{SD})$ & $0.96 \pm 0.93$ & $0.85 \pm 0.07$ & $0.85 \pm 0.08$ & $0.77 \pm 0.11$ & 0.15 \\
\hline cIMT mm (mean \pm SD) & $0.95 \pm 0.33$ & $0.91 \pm 0.32$ & $0.93 \pm 0.36$ & $1.21 \pm 0.31$ & 0.09 \\
\hline \multicolumn{6}{|l|}{ Lipid-Lowering Agents } \\
\hline Statin $n(\%)$ & $22(36.1 \%)$ & $14(36.8 \%)$ & $6(37.5 \%)$ & $2(28.6 \%)$ & \multirow{4}{*}{0.41} \\
\hline Statin + ezetimibe $n(\%)$ & $18(29.5 \%)$ & $9(23.7 \%)$ & $7(43.8 \%)$ & $2(28.6 \%)$ & \\
\hline Statin + fenofibrate $n(\%)$ & $8(13.1 \%)$ & $7(18.4 \%)$ & $1(6.3 \%)$ & 0 & \\
\hline Statin + ezetimibe + fenofibrate $n(\%)$ & $13(21.3 \%)$ & $8(21.1 \%)$ & $2(12.5 \%)$ & $3(42.9 \%)$ & \\
\hline DLCN Score (mean \pm SD) & $6.4 \pm 2.9$ & $5.9 \pm 2.5$ & $6.2 \pm 1.9$ & $9.6 \pm 4.9$ & $0.02 *$ \\
\hline
\end{tabular}

Legend: CHD—coronary heart disease, PAD—peripheral arterial disease, TC-total cholesterol, LDL-C-low-density cholesterol lipoprotein, HDL-C—high-density cholesterol lipoprotein, TG-triglycerides, hsCRP-high-sensitivity C-reactive protein, ECGelectrocardiogram, EF-ejection fraction, ABI-ankle-brachial index, cIMT—carotid intima-media thickness, DLCN-Dutch Lipid Clinic Network, ${ }^{*} p<0.05$. 
Table 2. $L D L R, P C S K 9$, and $A P O B$ variants identified in the Romania patients.

\begin{tabular}{|c|c|c|c|c|}
\hline Gene & Location & Nucleotide Change & Protein Change & Number of Carriers \\
\hline \multicolumn{5}{|c|}{ Pathogenic Variants } \\
\hline$L D L R$ & Exon 2 & c. $81 C>$ G & p.(Cys27Trp) & 2 \\
\hline$L D L R$ & Exon 4 & c. $502 \mathrm{G}>\mathrm{A}$ & p.(Asp168Asn) & 1 \\
\hline$L D L R$ & Exon 11 & c. $1618 \mathrm{G}>\mathrm{A}$ & p.(Ala540Thr) & 3 \\
\hline$L D L R$ & Exon 13-15 & $\begin{array}{c}\text { c. }\left(1845+1 \_1846-\right. \\
\text { 1)_(2311+1_2312-1)dup }\end{array}$ & $\mathrm{p}(?)$ & 1 \\
\hline \multicolumn{5}{|c|}{ Benign Variants } \\
\hline$L D L R$ & Exon 2 & c. $81 \mathrm{C}>\mathrm{T}$ & p. $($ Cys $27=)$ & 3 \\
\hline$L D L R$ & Exon 10 & c. $1413 \mathrm{~A}>\mathrm{G}$ & p.(Arg471=) & 15 \\
\hline$L D L R$ & Exon 11 & c. $1617 \mathrm{C}>\mathrm{T}$ & p. $($ Pro539=) & 3 \\
\hline$L D L R$ & Exon 12 & c. $1773 \mathrm{C}>\mathrm{T}$ & p.(Asn591=) & 12 \\
\hline$L D L R$ & Exon 13 & c. $1959 \mathrm{~T}>\mathrm{C}$ & p. $($ Val653=) & 12 \\
\hline$L D L R$ & Exon 15 & c. $2232 \mathrm{~A}>\mathrm{G}$ & p. $(\operatorname{Arg} 744=)$ & 14 \\
\hline$A P O B$ & Exon 26 & c. $10740 \mathrm{C}>\mathrm{T}$ & p. $($ Asn $3580=)$ & 3 \\
\hline \multicolumn{5}{|c|}{ Conflicting interpretations } \\
\hline$L D L R$ & Exon 3 & c. $211 G>A$ & p.(Gly71Arg) & 1 \\
\hline$L D L R$ & Exon 7 & c. $1060+7=$ & $\mathrm{p}(?)$ & 18 \\
\hline$L D L R$ & Intron 7 & c. $1060+10 \mathrm{G}>\mathrm{A}$ & $\mathrm{p}(?)$ & 8 \\
\hline PCSK9 & Exon 7 & c. $1026 \mathrm{~A}>\mathrm{G}$ & p. $(\mathrm{G} \ln 342=)$ & 6 \\
\hline
\end{tabular}

Legend: $L D L R$-low-density lipoprotein receptor, $A P O B$-apolipoprotein B; PCSK9-proprotein convertase subtilisin/kexin type 9 Variants were classified as pathogenic/likely pathogenic, benign/likely benign, and of conflicting interpretations according to ClinVar and Leiden Open Source Variation Database (LVOD).
A $C$ A $G$ A $T$ G $\stackrel{50}{S}$ G A A A A $A$ G A A A
A $C$ A $G$ A $T$ G $\stackrel{50}{S}$ G A A A A $A$ G A A A

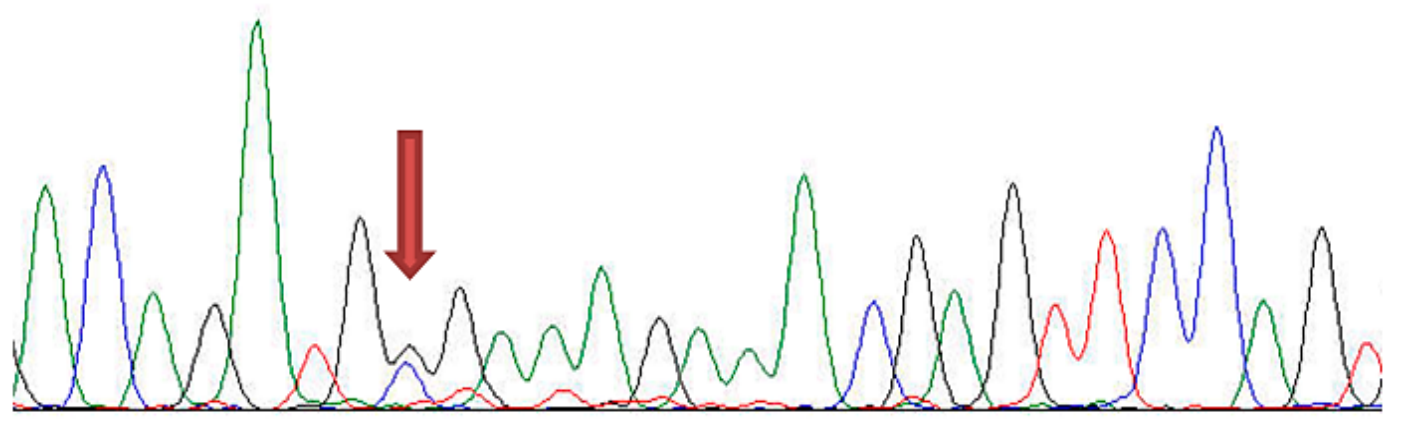

Figure 2. The Sanger sequencing electropherogram in patients H18 and H42: pathogen mutation of LDLR in exon 2 (c. $81 \mathrm{C}>\mathrm{G})$. 


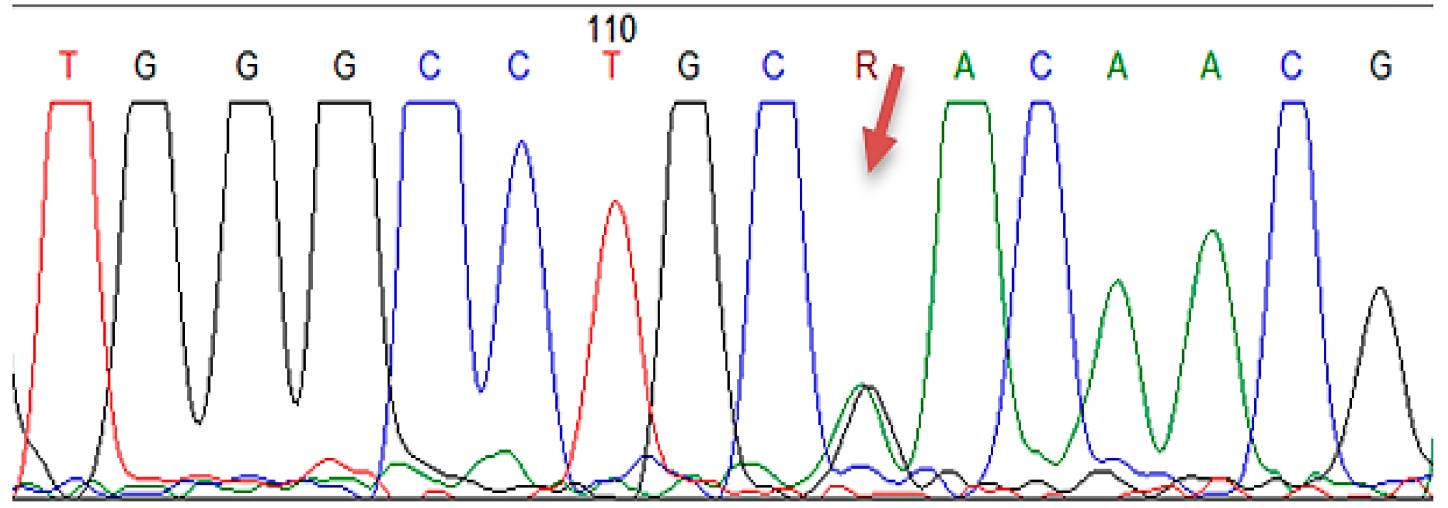

Figure 3. The Sanger sequencing electropherogram in patient H54: pathogen mutation of LDLR in exon 4 (c.502G > A).

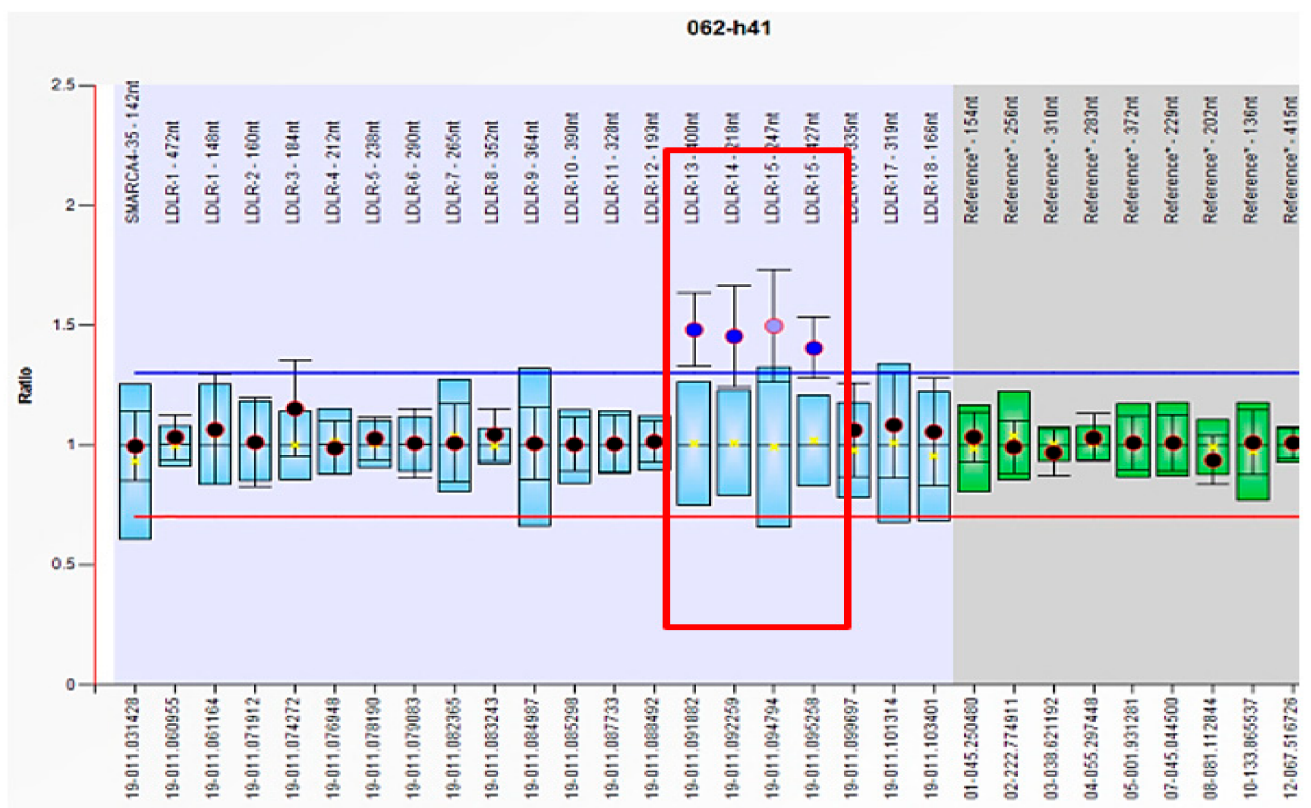

Figure 4. Multiplex ligation-dependent probe amplification (MLPA) method shows the duplication (ratio 1.5) in LDLR exons 13-15 in patient H41-exon numbers were shown above as "LDLR-number of exons" (the LDLR exon numbering uses the RefSeq transcript NM_000527.4). The reference probes were included for normalized probe-signal ratio. The arbitrary border (upper and lower) was placed \pm 0.3 from the reference sample median of probes.

According to the DLCN score, the FH patients without mutation had increased frequencies of probable $\mathrm{FH}$ and of defined $\mathrm{FH}$, compared to $\mathrm{FH}$ patients with benign/likely benign/conflicting classification or pathogen/likely pathogen mutations $\left(\chi^{2}(2)=5.7\right.$, $p=0.05$ ) (Figure 5). Depending on the DLCN score, the subjects with probable FH had $4.9 \%$ pathogenic/likely pathogenic mutations and $16.4 \%$ benign/ likely benign mutations, while the individuals with definite $\mathrm{FH}$ had $4.9 \%$ pathogenic/likely pathogenic mutations and $1.6 \%$ benign/likely benign mutations (Figure 5). 


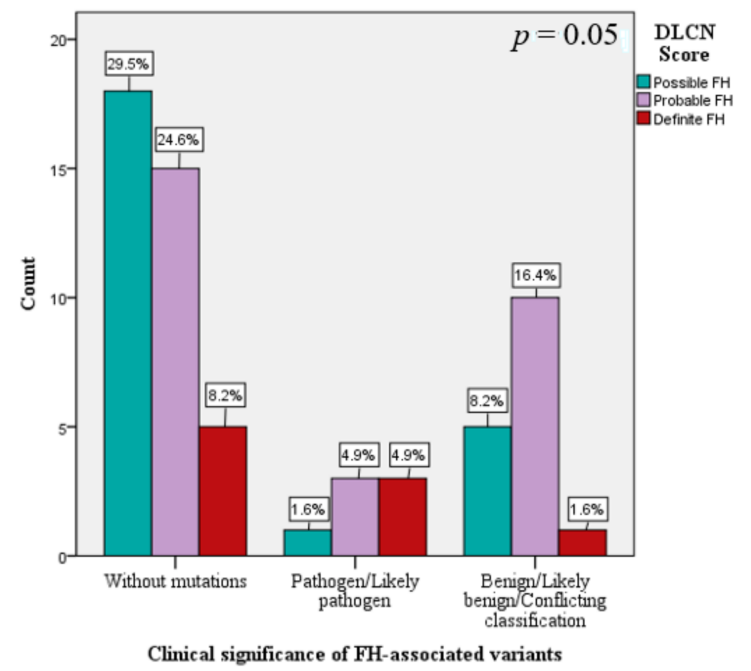

Figure 5. The frequency of mutations and DLCN score.

\subsection{The New ASCVD in Patients with FH Based on LDLR, APOB, and PCSK9 Mutations}

The seven patients with pathogenic mutations advertised new cardiovascular events as follows: three patients with c.1618G > A in homozygous state had the acute coronary syndrome, two with c.81C > G in the heterozygous state had a stroke and peripheral artery disease (PAD) and one with c.502G > A and dup ex13-15 had a stroke (Table S1). Additionally, among the 16 patients with benign/likely benign mutations, only eight had new ASCVD: three patients experienced a stroke, three had PAD, and two patients had acute coronary syndrome (Table S1).

Patients with pathogenic/likely pathogenic mutations and with ASCVD had elevated TC, LDL-C levels throughout the follow up, with cIMT values increased at 12 and 36 months, whereas the ejection fraction (EF) and HDL-C values were significantly declined at 36 months, compared to patients with benign mutations and ASCVD or mutation-free subjects with ASCVD (Table 3). The FH patients with ASCVD but no mutations recorded elevated hsCRP levels throughout the follow-up period, compared to FH patients with ASCVD and mutations. Moreover, ankle-brachial index (ABI) values were decreased in FH patients with ASCVD and benign mutations, compared to patients with ASCVD without mutations (Table 3).

Furthermore, in FH patients, following the multiple regression, pathogenic/likely pathogenic mutations were significant predictors of the new cardiovascular events $(\mathrm{OR}=4.81,95 \% \mathrm{CI}: 1.26-18.32, p=0.02)$ (Table 4). Interestingly, lipid-lowering drugs did not act as a protective factor for the new cardiovascular disease in the case of these patients $(p=0.72)$ (Table 4$)$.

For FH patients with $L D L R, A P O B$, and PCSK9 mutations, the time interval for the occurrence of new cardiovascular events was significantly decreased, compared to FH patients without mutations (14 months vs. 27 months, $p=0.001$ ) (Figure 6a). Following the stratification according to the clinical significance of FH-associated mutations, there were no significant differences in the time interval for new ASCVD occurrence (Figure 6b). 
Table 3. The characteristics of FH patients according to cardiovascular events.

\begin{tabular}{|c|c|c|c|c|c|c|}
\hline \multirow[b]{2}{*}{ Characteristics } & \multicolumn{6}{|c|}{ Patients with FH } \\
\hline & $\begin{array}{l}\text { ASCVD- } \\
\text { Mutation- }\end{array}$ & $\begin{array}{l}\text { ASCVD+ } \\
\text { Mutation- }\end{array}$ & $\begin{array}{c}\text { ASCVD- } \\
\text { Benign/Likely } \\
\text { Benign } \\
\text { Mutation+ }\end{array}$ & $\begin{array}{c}\text { ASCVD+ } \\
\text { Benign/Likely } \\
\text { Benign } \\
\text { Mutation+ }\end{array}$ & $\begin{array}{c}\text { ASCVD+ } \\
\text { Pathogenic/Likely } \\
\text { PATHOGENIC } \\
\text { Mutation+ }\end{array}$ & $p$ \\
\hline$n(\%)$ & $25(41 \%)$ & $13(21.31 \%)$ & $8(13.1 \%)$ & $8(13.1 \%)$ & $7(11.5 \%)$ & \\
\hline TC baseline mg/dL (median $\pm \mathrm{IQR})$ & $312.7 \pm 20.4$ & $352.8 \pm 63.2$ & $323.9 \pm 26.9$ & $352.6 \pm 42.2$ & $445.6 \pm 203.9$ & $0.01 *$ \\
\hline TC 12 mo mg/dL (median \pm IQR) & $249.1 \pm 17.9$ & $284.5 \pm 51.8$ & $261 \pm 13.9$ & $278.5 \pm 44.2$ & $310.3 \pm 63.5$ & 0.009 * \\
\hline TC 24 mo mg/dL (median \pm IQR) & $227.7 \pm 19.3$ & $254.8 \pm 47.4$ & $236.1 \pm 12.9$ & $256.6 \pm 29.8$ & $278.7 \pm 57.9$ & $0.01 *$ \\
\hline TC 36 mo mg/dL (median \pm IQR) & $210.8 \pm 17.1$ & $230.9 \pm 14.3$ & $229.3 \pm 12.2$ & $245.6 \pm 29.6$ & $271.9 \pm 53.3$ & $0.001 *$ \\
\hline $\mathrm{LDL}-\mathrm{C}$ baseline $\mathrm{mg} / \mathrm{dL}($ mean $\pm \mathrm{SD})$ & $233.4 \pm 29.1$ & $270.8 \pm 62.5$ & $240.1 \pm 29.2$ & $257.8 \pm 54.7$ & $309.7 \pm 78.7$ & 0.15 \\
\hline LDL-C 12 mo mg/dL (mean \pm SD) & $163.6 \pm 21.4$ & $204.4 \pm 50.9$ & $166.6 \pm 13.3$ & $195.4 \pm 52.9$ & $234.1 \pm 66.7$ & 0.002 * \\
\hline LDL-C 24 mo mg/dL (mean \pm SD) & $137.4 \pm 17.8$ & $167.5 \pm 47.6$ & $139.6 \pm 14.2$ & $168.5 \pm 39.7$ & $202.4 \pm 59.7$ & 0.003 * \\
\hline LDL-C 36 mo mg/dL (mean \pm SD) & $113.1 \pm 17.3$ & $146.5 \pm 11.6$ & $131.1 \pm 14.9$ & $159.1 \pm 35.1$ & $189.3 \pm 56.5$ & 0.001 * \\
\hline HDL-C baseline $\mathrm{mg} / \mathrm{dL}$ (median $\pm \mathrm{IQR}$ ) & $51.3 \pm 12.5$ & $42.4 \pm 9.8$ & $54.5 \pm 16.4$ & $55.5 \pm 19.9$ & $43.1 \pm 7.9$ & 0.14 \\
\hline HDL-C 12 mo mg/dL (median \pm IQR) & $61.7 \pm 8.9$ & $53.9 \pm 9.8$ & $64.3 \pm 12.1$ & $64.4 \pm 12.4$ & $54.9 \pm 10.1$ & 0.19 \\
\hline HDL-C 24 mo mg/dL (median \pm IQR) & $67.4 \pm 7.9$ & $62.4 \pm 7.1$ & $71.1 \pm 6.8$ & $68.4 \pm 9.4$ & $59.4 \pm 10.8$ & $0.05 *$ \\
\hline HDL-C 36 mo mg/dL (median \pm IQR) & $75.1 \pm 8.2$ & $61.6 \pm 7.7$ & $73.1 \pm 7.9$ & $65.1 \pm 4.8$ & $58.3 \pm 5.8$ & 0.001 * \\
\hline TG baseline mg/dL (mean \pm SD) & $166.8 \pm 90.9$ & $203.7 \pm 93.3$ & $204.1 \pm 128.1$ & $140.1 \pm 80.2$ & $152.4 \pm 47.9$ & 0.19 \\
\hline TG 12 mo mg/dL (mean \pm SD) & $124.2 \pm 48.8$ & $142.4 \pm 51.3$ & $151.6 \pm 69.4$ & $109.1 \pm 39.7$ & $124.7 \pm 29.7$ & 0.26 \\
\hline TG 24 mo mg/dL (mean \pm SD) & $116.5 \pm 31.2$ & $124.9 \pm 30.4$ & $125.1 \pm 36.3$ & $106.8 \pm 34.3$ & $122.4 \pm 30.5$ & 0.41 \\
\hline TG 36 mo mg/dL (mean \pm SD) & $120.6 \pm 22.3$ & $123.1 \pm 22.3$ & $126.1 \pm 28.5$ & $107.1 \pm 33.1$ & $121.7 \pm 25.4$ & 0.25 \\
\hline hsCRP baseline $\mathrm{mg} / \mathrm{L}($ mean $\pm \mathrm{SD})$ & $5.1 \pm 1.9$ & $7.3 \pm 1.8$ & $6.1 \pm 2.7$ & $6.7 \pm 1.6$ & $7.1 \pm 2.5$ & $0.02 *$ \\
\hline hsCRP 12 mo mg/L (mean \pm SD) & $4.1 \pm 1.8$ & $6.1 \pm 1.3$ & $4.8 \pm 1.9$ & $5.7 \pm 1.6$ & $5.8 \pm 2.4$ & $0.02 *$ \\
\hline hsCRP 24 mo mg / L (mean \pm SD) & $3.5 \pm 1.4$ & $5.3 \pm 1.3$ & $4.1 \pm 1.6$ & $4.4 \pm 1.7$ & $4.7 \pm 2.3$ & $0.01 *$ \\
\hline hsCRP 36 mo mg/L (mean \pm SD) & $0.6 \pm 0.2$ & $7.2 \pm 1.2$ & $2.4 \pm 2.7$ & $5.9 \pm 1.1$ & $6.7 \pm 1.8$ & 0.001 * \\
\hline EF baseline \% (mean \pm SD) & $56.4 \pm 7.6$ & $48.7 \pm 10.5$ & $53.8 \pm 8.6$ & $49.4 \pm 10.8$ & $53.3 \pm 12.9$ & 0.13 \\
\hline $\mathrm{EF} 12 \mathrm{mo} \%($ mean $\pm \mathrm{SD})$ & $55.4 \pm 6.6$ & $48.1 \pm 9.6$ & $52.5 \pm 7.6$ & $48.8 \pm 8.4$ & $50.7 \pm 14.8$ & 0.11 \\
\hline $\mathrm{EF} 24 \mathrm{mo} \%($ mean $\pm \mathrm{SD})$ & $54.8 \pm 6.3$ & $43.1 \pm 10.7$ & $52.5 \pm 7.6$ & $47.5 \pm 8.1$ & $44.3 \pm 16.2$ & $0.006 *$ \\
\hline $\mathrm{EF} 36 \mathrm{mo} \%($ mean $\pm \mathrm{SD})$ & $53.8 \pm 6.7$ & $41.2 \pm 12.1$ & $51.9 \pm 7.5$ & $45 \pm 10.4$ & $37.9 \pm 17.3$ & $0.002 *$ \\
\hline ABI baseline (mean \pm SD) & $0.86 \pm 0.06$ & $0.81 \pm 0.06$ & $0.89 \pm 0.08$ & $0.79 \pm 0.06$ & $0.78 \pm 0.11$ & 0.01 * \\
\hline $\mathrm{ABI} 12$ mo (mean $\pm \mathrm{SD})$ & $0.89 \pm 0.05$ & $0.85 \pm 0.05$ & $0.91 \pm 0.06$ & $0.81 \pm 0.06$ & $0.81 \pm 0.08$ & $0.001 *$ \\
\hline $\mathrm{ABI} 24$ mo (mean $\pm \mathrm{SD})$ & $0.94 \pm 0.07$ & $0.89 \pm 0.71$ & $0.94 \pm 0.08$ & $0.84 \pm 0.13$ & $0.85 \pm 0.07$ & 0.004 * \\
\hline $\mathrm{ABI} 36$ mo (mean $\pm \mathrm{SD})$ & $0.94 \pm 0.04$ & $0.82 \pm 0.11$ & $0.94 \pm 0.03$ & $0.81 \pm 0.09$ & $0.83 \pm 0.09$ & $0.001 *$ \\
\hline cIMT baseline $\mathrm{mm}($ mean $\pm \mathrm{SD})$ & $0.82 \pm 0.28$ & $1.08 \pm 0.32$ & $0.89 \pm 0.36$ & $1.13 \pm 0.31$ & $1.04 \pm 0.42$ & 0.03 * \\
\hline cIMT 12 mo mm (mean \pm SD) & $0.75 \pm 0.23$ & $1.03 \pm 0.25$ & $0.81 \pm 0.31$ & $1.05 \pm 0.24$ & $1.06 \pm 0.31$ & $0.002 *$ \\
\hline cIMT 24 mo mm (mean \pm SD) & $0.74 \pm 0.25$ & $0.99 \pm 0.31$ & $0.75 \pm 0.29$ & $0.96 \pm 0.31$ & $0.94 \pm 0.36$ & $0.01 *$ \\
\hline cIMT 36 mo mm (mean \pm SD) & $0.73 \pm 0.15$ & $1.07 \pm 0.18$ & $0.76 \pm 0.12$ & $1.08 \pm 0.21$ & $1.13 \pm 0.15$ & $0.001 *$ \\
\hline
\end{tabular}

Legend: ASCVD- atherosclerotic cardiovascular disease, TC-total cholesterol, LDL-C—low-density cholesterol lipoprotein, HDL-C-highdensity cholesterol lipoprotein, TG—triglycerides, hsCRP-high-sensitivity C-reactive protein, EF-ejection fraction, ABI-ankle-brachial index, cIMT—carotid intima-media thickness, mo-months * $p<0.05$. 
Table 4. Independent factors for cardiovascular events in FH patients.

\begin{tabular}{|c|c|c|c|c|c|c|c|c|}
\hline \multirow{2}{*}{ Variable } & \multirow{2}{*}{ B } & \multirow{2}{*}{ SE } & \multirow{2}{*}{ Wald } & \multirow{2}{*}{ df } & \multirow{2}{*}{$p$} & \multirow{2}{*}{ OR } & \multicolumn{2}{|c|}{$95.0 \% \mathrm{CI}$ for $\mathrm{HR}$} \\
\hline & & & & & & & Lower & Upper \\
\hline \multirow{3}{*}{$\begin{array}{l}\text { Type of mutations } \\
\text { Pathogenic Mutations } \\
\text { Benign Mutations }\end{array}$} & & & 5.41 & 2 & 0.05 * & & & \\
\hline & 1.57 & 0.68 & 5.29 & 1 & $0.02 *$ & 4.81 & 1.26 & 18.32 \\
\hline & 0.37 & 0.48 & 0.61 & 1 & 0.44 & 1.45 & 0.57 & 3.73 \\
\hline EF baseline & 0.01 & 0.03 & 0.05 & 1 & 0.82 & 1.01 & 0.95 & 1.08 \\
\hline cIMT baseline & 0.33 & 1.12 & 0.08 & 1 & 0.77 & 1.39 & 0.15 & 13.29 \\
\hline ABI baseline & -3.96 & 4.74 & 0.69 & 1 & 0.41 & 0.02 & 0.001 & 20.73 \\
\hline hsCRP baseline & 0.16 & 0.13 & 1.51 & 1 & 0.22 & 1.18 & 0.91 & 1.53 \\
\hline LDL-C baseline & 0.04 & 0.08 & 0.33 & 1 & 0.57 & 1.01 & 0.99 & 1.02 \\
\hline \multirow{3}{*}{$\begin{array}{c}\text { DLCN Score } \\
\text { Possible FH } \\
\text { Probable FH } \\
\text { Definite FH }\end{array}$} & ref & ref & $\begin{array}{c}1.69 \\
\text { ref }\end{array}$ & $\begin{array}{c}2 \\
\text { ref }\end{array}$ & $\begin{array}{c}0.43 \\
\text { ref }\end{array}$ & ref & ref & ref \\
\hline & 0.48 & 0.66 & 0.53 & 1 & 0.47 & 1.62 & 0.45 & 5.87 \\
\hline & -0.39 & 1.51 & 0.07 & 1 & 0.79 & 0.67 & 0.04 & 12.94 \\
\hline Simon Broome score & 0.56 & 0.73 & 0.59 & 1 & 0.44 & 1.75 & 0.42 & 7.37 \\
\hline \multirow{4}{*}{$\begin{array}{c}\text { Lipid-lowering } \\
\text { DrugsStatin } \\
\text { Statin + Ezetimibe } \\
\text { Statin + Fenofibrate } \\
\text { Statin + Fenofibrate + } \\
\text { Ezetimibe }\end{array}$} & ref & ref & $\begin{array}{l}1.35 \\
\text { ref }\end{array}$ & $\begin{array}{c}3 \\
\text { ref }\end{array}$ & $\begin{array}{c}0.72 \\
\text { ref }\end{array}$ & ref & ref & ref \\
\hline & 0.15 & 0.61 & 0.06 & 1 & 0.81 & 1.17 & 0.35 & 3.84 \\
\hline & 0.46 & 0.76 & 0.37 & 1 & 0.54 & 1.59 & 0.36 & 7.09 \\
\hline & -0.44 & 0.67 & 0.44 & 1 & 0.51 & 0.64 & 0.18 & 2.36 \\
\hline
\end{tabular}

Legend: LDL-C-low-density cholesterol lipoprotein, hsCRP—high-sensitivity C-reactive protein, EF-ejection fraction, ABI-ankle-brachial index, DLCN-Dutch Lipid Clinic Network, cIMT—carotid intima-media thickness, OR odd ratio * $p<0.05$.

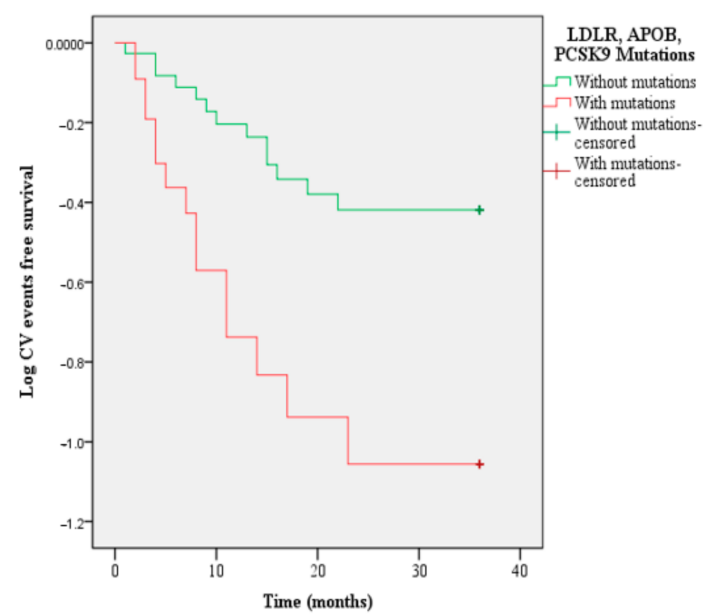

(a)

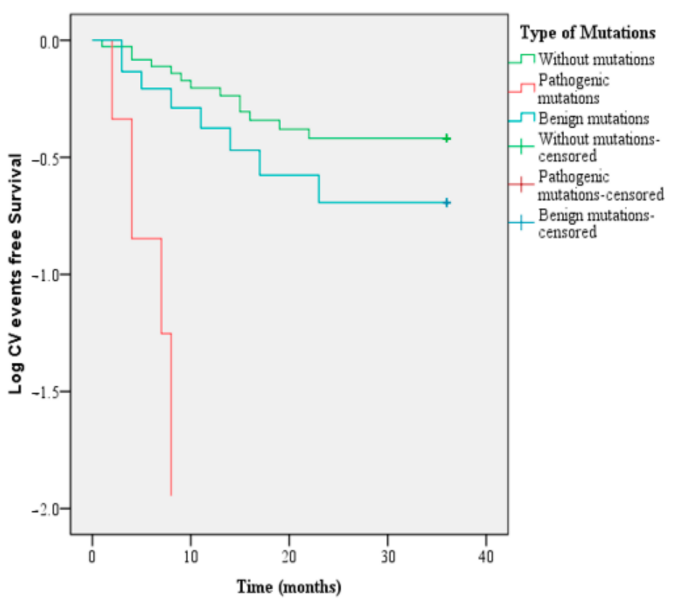

(b)

Figure 6. Kaplan-Meier for ASCVD depending on (a) LDLR, APOB, and PCSK9 mutations and (b) clinical significance of FH-associated variants and time interval for the occurrence of new CV events. Legend: LDLR-low-density cholesterol lipoprotein receptor, APOB-apolipoprotein B, PCSK9-proprotein convertase subtilisin/kexin type 9, CV—cardiovascular.

\section{Discussion}

In this study, two techniques were used to identify mutations in patients with a clinical diagnosis of FH-multiplex ligation-dependent probe amplification (MLPA) and Sanger sequencing. Since DNA sequencing cannot detect large gene rearrangements, the MLPA approach was performed [30]. The Leiden Open Source Variation Database (LOVD) is a virtual space of genetic variants, comprising 1707 unique LDLR variants since 2016, 
which can be accessed by all physicians in the preclinical and clinical fields [31]. However, ClinVar is a resource funded by the National Center for Biotechnology Information (NCBI), which provides a centralized database for archiving clinically relevant variants for many Mendelian pathologies, including FH [31,32]. ClinVar is a comprehensive approach to show the molecular data about patients, including many interconnected resources to improve the interpretation of the variants [31,32]. Both ClinVar and LOVD show the mutation type identified by the geneticist-pathogen/likely pathogen, benign/likely benign, or conflicting interpretations. The term "pathogen" means affecting the protein function causing disease, while "benign" indicates the lack of impairment of protein function, without causing disease [33]. However, the applicability of the term "likely" is limited to variants in which the data support a high probability of being pathogenic $(>90 \%)$ or a high probability of being benign $(>90 \%)$, without providing a quantitative definition of these aspects [33].

In the current study, the mutation rate registered was $37.7 \%, 23$ patients with mutations being identified -7 patients with pathogenic/likely pathogenic mutations and 16 with polymorphisms. Regarding the pathogenic/likely pathogenic mutations, it is striking that the same mutations were targeted at exons 2,4 , and 11 of the LDLR gene. Therefore, further studies that include a large number of patients are recommended, targeting the identification of the genetic spectrum of $\mathrm{FH}$ in Romania and other populations. The difference in plasma lipid levels, even in patients bearing the same mutation, could be induced by other genetic and /or environmental factors, and the possibility of a second mutation in the LDLR gene should be considered [34].

Variants of the LDLR, APOB, PCSK9 genes, similar to those included in the study conducted in this region of Romania, were presented by several other authors, as indicated in Table $5[1-5,7-11,15,16,30,34-44]$.

In our study, we identified two patients with the c.81C $>$ G mutation in exon 2 of LDLR gene with heterozygous form, which had two new $\mathrm{CV}$ events during the follow up-stroke and PAD. Mollaki et al. showed in a cohort study that c.1646G > A and c.1285G > A were associated with high lipid levels compared to c.858C $>$ A and c.81C $>$ G, which cause a milder phenotype [30]. The genotype-to-phenotype correlations revealed that receptor defective mutations cause lowered lipid levels than receptor-negative mutations of LDLR, c. $81 \mathrm{C}>\mathrm{G}$ being considered a defective mutation of $L D L R$ [30]. The c.81C $>\mathrm{G}$ mutation in exon 2 of the LDLR gene was identified in Greece in 37 patients [30], in Switzerland in one patient [15], and in Slovakia in two patients [3] (Table 5), while in our study it was detected in two patients with heterozygous status.

The c.502G > A mutation in exon 4 of $L D L R$ gene was observed in few studies-in the UK in one patient [45], in Spain in one patient [46], in the Czech Republic in one subject [47], and in Canada in one subject [1] (Table 5), while in our study we identified the same mutation in one patient with new ASCVD (stroke).

The c.1618G > A mutation in exon 11 of the LDLR gene was described in the study carried out by Chiou et al. in one patient from Taiwan [44], in Brazil in one patient, and in the study conducted by Jannes et al. [4], in Australia in one patient [11], in the UK in one patient [35], in Japan in one patient [34], in Greece in one patient [38], in Spain in two patients [16], and in Germany in two patients [37] (Table 5). In this study conducted in Romania, three patients with the same mutation (c.1618G > A) with homozygous status in the exon 11 of $L D L R$ gene have been identified. 
Table 5. Characteristics of different studies for genotypes of the FH patients.

\begin{tabular}{|c|c|c|c|c|c|c|c|}
\hline Locality & Country & $\begin{array}{l}\text { Diagnostic } \\
\text { Criteria }\end{array}$ & $\begin{array}{l}\text { Number } \\
\text { of } \\
\text { Patients }\end{array}$ & $\begin{array}{l}\text { Number of } \\
\text { Patients with } \\
\text { Mutations }\end{array}$ & $\begin{array}{c}\text { Technique- Molecular } \\
\text { Analysis }\end{array}$ & Gene & $\begin{array}{l}\text { Number } \\
\text { of Detected } \\
\text { Mutations }\end{array}$ \\
\hline \multirow{8}{*}{$\begin{array}{l}\text { Western } \\
\text { Europe }\end{array}$} & $\begin{array}{l}\text { Italy } \\
\text { [2] }\end{array}$ & DLCN & 1018 & 94 & $\begin{array}{c}\text { MLPAN } \\
\text { orthern blot analysis and } \\
\text { RT-PCR amplification } \\
\text { In silico analysis }\end{array}$ & $\begin{array}{l}\text { LDLR } \\
\text { APOB } \\
\text { PCSK9 }\end{array}$ & $\begin{array}{l}984 \text { LDLR } \\
22 \text { APOB } \\
2 \text { PCSK9 }\end{array}$ \\
\hline & $\begin{array}{l}\text { Switzerland } \\
\text { [15] }\end{array}$ & $\begin{array}{l}\text { LDL-C 95th } \\
\text { percentile }\end{array}$ & 94 & NA & $\begin{array}{c}\text { NGS (Illumina) } \\
\text { Sanger sequencing }\end{array}$ & $\begin{array}{l}\angle D L R \\
A P O B \\
\text { PCSK9 }\end{array}$ & $\begin{array}{l}43 \text { LDLR } \\
5 \text { APOB3 } \\
6 \text { PCSK9 }\end{array}$ \\
\hline & $\begin{array}{c}\text { UK-1 } \\
{[45]}\end{array}$ & SB criteria & 791 & 134 & SSCP analysis & $L D L R$ & $51 L D L R$ \\
\hline & $\begin{array}{c}\text { UK-2 } \\
{[35]}\end{array}$ & SB criteria & 280 & $\begin{array}{c}171 \mathrm{He} \mathrm{FH} \\
\text { patients } \\
28 \mathrm{Ho} / \\
\text { compound He }\end{array}$ & $\begin{array}{c}\text { MLPA } \\
\text { sequencing of amplified } \\
\text { fragments of genomic } \\
\text { DNA or mRNA }\end{array}$ & $\begin{array}{l}\text { LDLR } \\
\text { LDLRAP1 } \\
P C S K 9 \\
A P O B\end{array}$ & $\begin{array}{l}98 \text { LDLR } \\
2 \text { PCSK9 } \\
5 \text { LDLRAP1 } \\
14 \text { APOB }\end{array}$ \\
\hline & $\begin{array}{l}\text { UK-3 } \\
{[36]}\end{array}$ & $\begin{array}{l}\text { SB FH register } \\
\text { SB criteria }\end{array}$ & 48 & 14 & $\begin{array}{c}\text { MLPA } \\
\text { NGS (Illumina) } \\
\text { Sanger sequencing }\end{array}$ & $\begin{array}{c}\text { LDLR } \\
\text { LDLRAP1 } \\
\text { PCSK9 } \\
\text { APOB }\end{array}$ & $\begin{array}{l}17 \text { LDLR } \\
1 \text { LDLRAP1 } \\
2 \text { PCSK9 } \\
3 \text { APOB }\end{array}$ \\
\hline & $\begin{array}{c}\text { Spain } \\
{[16]}\end{array}$ & $\begin{array}{l}\text { Spanish FH } \\
\text { Registry }\end{array}$ & 476 & 329 & SSCP analysis & $\angle D L R A P O B$ & $\begin{array}{c}116 \text { LDLR } \\
4 A P O B\end{array}$ \\
\hline & $\begin{array}{l}\text { Germany } \\
\text { [37] }\end{array}$ & $\begin{array}{l}\text { LDL-C 90th } \\
\text { percentile }\end{array}$ & 162 & 27 & $\begin{array}{c}\text { MLPA } \\
\text { Direct sequencing on } \\
\text { LDLR gene }\end{array}$ & $L D L R$ & $24 L D L R$ \\
\hline & $\begin{array}{c}\text { Portugal } \\
\text { (Azores } \\
\text { Island) } \\
\text { [5] }\end{array}$ & SB & 33 & 33 & $\begin{array}{c}\text { LIPOchip }{ }^{\circledR} \text { Array version } \\
7 \text { (DNA array) } \\
\text { Direct sequencing for } \\
\text { exons } 2-6\end{array}$ & $L D L R$ & $18 L D L R$ \\
\hline \multirow{7}{*}{$\begin{array}{l}\text { Central and } \\
\text { Eastern } \\
\text { Europe }\end{array}$} & $\begin{array}{c}\text { Slovakia } \\
\text { [3] }\end{array}$ & $\begin{array}{c}\text { LDL-C 95th } \\
\text { percentile }+\mathrm{HCH} \\
\text { in family }\end{array}$ & 359 & $\begin{array}{l}16 \text { for APOB } \\
164 \text { for LDLR }\end{array}$ & $\begin{array}{c}\text { TaqMan SNP Genotyping } \\
\text { Assay ID } \\
\text { Bidirectional sequencing } \\
\text { on LDLR gene } \\
\text { MLPA }\end{array}$ & $\begin{array}{l}\angle D L R \\
A P O B\end{array}$ & $\begin{array}{l}54 \text { LDLR } \\
1 A P O B\end{array}$ \\
\hline & $\begin{array}{l}\text { Greece-1 } \\
\text { [38] }\end{array}$ & $\begin{array}{l}\text { LDL-C 95th } \\
\text { percentile } \\
\text { CVD history } \\
\text { CVD family } \\
\text { history tendon } \\
\text { xanthomas }\end{array}$ & 183 & 78 & DGGE analysis & $\begin{array}{l}\angle D L R \\
A P O B\end{array}$ & $\begin{array}{l}17 L D L R \\
0 A P O B\end{array}$ \\
\hline & $\begin{array}{l}\text { Greece-2 } \\
{[30]}\end{array}$ & HeFH & 561 & 140 & $\begin{array}{l}\text { DNA sequencing of the } \\
\text { LDLR gene }\end{array}$ & $L D L R$ & $26 L D L R$ \\
\hline & $\begin{array}{c}\text { Russia } 1 \\
\text { [39] }\end{array}$ & DLCN & 80 & 80 & $\begin{array}{l}\text { Sanger sequencing } \\
\text { In silico analysis }\end{array}$ & $\begin{array}{l}\angle D L R \\
A P O B\end{array}$ & $\begin{array}{l}12 L D L R \\
0 A P O B\end{array}$ \\
\hline & $\begin{array}{c}\text { Russia } 2 \\
\text { [40] }\end{array}$ & $\begin{array}{l}\text { LDL-C 95th } \\
\text { percentile } \\
\text { CVD history } \\
\text { CVD family } \\
\text { history tendon } \\
\text { xanthomas }\end{array}$ & 45 & 24 & $\begin{array}{l}\text { Automated DNA } \\
\text { sequencing }\end{array}$ & $\begin{array}{l}L D L R \\
A P O B\end{array}$ & $\begin{array}{l}21 L D L R \\
0 A P O B\end{array}$ \\
\hline & $\begin{array}{l}\text { Poland-1 } \\
\text { [41] }\end{array}$ & $\begin{array}{l}\text { LDL-C 90th } \\
\text { percentile }\end{array}$ & 30 families & 17 families & $\begin{array}{c}\text { SSCP analysis } \\
\text { sequencing of } \\
\text { polymerase chain } \\
\text { reaction } \\
\text { restriction enzyme } \\
\text { patterns on Southern } \\
\text { blots and long-PCR }\end{array}$ & $\begin{array}{l}L D L R \\
A P O B\end{array}$ & $\begin{array}{l}11 \angle D L R \\
1 A P O B\end{array}$ \\
\hline & $\begin{array}{c}\text { Poland-2 } \\
\text { [9] }\end{array}$ & SB & 161 & 40 & $\begin{array}{l}\text { High resolution melt } \\
\text { Direct sequencing } \\
\text { MLPA }\end{array}$ & $\begin{array}{l}L D L R \\
A P O B\end{array}$ & $\begin{array}{l}39 \text { LDLR } \\
1 A P O B\end{array}$ \\
\hline
\end{tabular}


Table 5. Cont.

\begin{tabular}{|c|c|c|c|c|c|c|c|}
\hline Locality & Country & $\begin{array}{l}\text { Diagnostic } \\
\text { Criteria }\end{array}$ & $\begin{array}{c}\text { Number } \\
\text { of } \\
\text { Patients }\end{array}$ & $\begin{array}{l}\text { Number of } \\
\text { Patients with } \\
\text { Mutations }\end{array}$ & $\begin{array}{c}\text { Technique- Molecular } \\
\text { Analysis }\end{array}$ & Gene & $\begin{array}{c}\text { Number } \\
\text { of Detected } \\
\text { Mutations }\end{array}$ \\
\hline & $\begin{array}{c}\text { Czech } \\
\text { Republic } \\
\text { [47] }\end{array}$ & $\begin{array}{l}\text { personal history } \\
\text { and/or family } \\
\text { history of } \\
\text { premature CHD } \\
\text { elevated TC, } \\
\text { LDL95th } \\
\text { percentile }\end{array}$ & 3914 & 1296 & $\begin{array}{c}\text { denaturing } \\
\text { high-performance liquid } \\
\text { chromatography } \\
\text { (dHPLC) } \\
\text { PCR-RFLP } \\
\text { Sanger sequencing } \\
\text { MLPA }\end{array}$ & $\begin{array}{l}L D L R \\
A P O B\end{array}$ & $\begin{array}{l}864 \text { LDLR } \\
32 A P O B\end{array}$ \\
\hline \multirow{10}{*}{ Worldwide } & $\begin{array}{l}\text { Canada } \\
\text { [1] }\end{array}$ & $\begin{array}{l}\text { DLCN } \\
\text { The British } \\
\text { Columbia FH } \\
\text { Registry }\end{array}$ & 626 & 275 & $\begin{array}{c}\text { NGS } \\
\text { (Illumina) }\end{array}$ & $\begin{array}{c}\text { LDLR } \\
\text { APOB } \\
\text { PCSK9 } \\
\text { LDLRAP1 }\end{array}$ & $\begin{array}{l}131 \text { unique } \\
\text { FH-causing } \\
\text { SNVs } \\
38 \mathrm{CNV} \\
\text { LDLR } \\
0 \mathrm{CNV} \\
\text { PCSK9, } \\
\text { APOB }\end{array}$ \\
\hline & $\begin{array}{c}\text { Brazil } \\
\text { [4] }\end{array}$ & $\begin{array}{l}\text { DLCN } \\
\text { SB }\end{array}$ & 248 & 125 & MLPA & $\begin{array}{l}\text { LDLR } \\
\text { APOB } \\
\text { PCSK9 }\end{array}$ & $\begin{array}{l}71 \text { LDLR } \\
2 \text { APOB } \\
0 \text { PCSK9 } \\
\end{array}$ \\
\hline & $\begin{array}{l}\text { Colombia } \\
\text { [42] }\end{array}$ & MedPed & 24 families & NA & Sanger sequencing & $L D L R$ & $\begin{array}{l}18 L D L R \\
3 \text { pathogenic } \\
\text { LDLR }\end{array}$ \\
\hline & $\begin{array}{c}\text { Australia } \\
\text { [11] }\end{array}$ & $\begin{array}{l}\text { mutations } \\
\text { previously } \\
\text { determined }\end{array}$ & 30 & NA & $\begin{array}{c}\text { Ion Torrent Personal } \\
\text { Genome Machine (PGM) } \\
\text { sequencing } \\
\text { Sanger sequencing } \\
\text { MLPA }\end{array}$ & $L D L R$ & $2179 L D L R$ \\
\hline & $\begin{array}{l}\text { Malaysia } \\
\text { [10] }\end{array}$ & SB & 164 & 117 & $\begin{array}{c}\text { Denaturing } \\
\text { High-Performance } \\
\text { Liquid Chromatography } \\
\text { MLPA } \\
\text { In silico analyses of } \\
\text { variant effects }\end{array}$ & $L D L R$ & $\begin{array}{l}8 \text { mutation } \\
L D L R 21 \\
\text { variants }\end{array}$ \\
\hline & $\begin{array}{c}\text { Sri Lanka } \\
\text { [7] }\end{array}$ & $\begin{array}{l}\text { Modified SB } \\
\text { DCLN }\end{array}$ & 27 & 5 & Sanger sequencing & $L D L R$ & $\begin{array}{l}4 \text { variants He } \\
1 \text { mutation } \\
\text { He } \\
\text { compound }\end{array}$ \\
\hline & $\begin{array}{c}\text { Saudi Arabia } \\
\text { [43] }\end{array}$ & DLCN & 2 & 2 & Sanger sequencing & $\begin{array}{l}\text { LDLR } \\
\text { APOB } \\
\text { PCSK9 }\end{array}$ & $\begin{array}{l}2 \text { LDLR } \\
\text { mutations } \\
0 \text { APOB } \\
0 \text { PCSK9 }\end{array}$ \\
\hline & $\begin{array}{c}\text { Iran } \\
{[8]}\end{array}$ & SB & 80 & NA & $\begin{array}{c}\text { ARMS-PCR } \\
\text { PCR- RFLP assay }\end{array}$ & $\begin{array}{l}\text { LDLR } \\
\text { APOB } \\
\text { PCSK9 }\end{array}$ & $\begin{array}{c}2 \text { LDLR } \\
\text { mutations } \\
6 \text { LDLR poly- } \\
\text { morphism } \\
0 \text { APOB } \\
0 \text { PCSK9 }\end{array}$ \\
\hline & $\begin{array}{c}\text { Taiwan } \\
\text { [44] }\end{array}$ & SB & 125 & 76 & $\begin{array}{l}\text { Microarray resequencing } \\
\text { Sanger sequencing }\end{array}$ & $\begin{array}{l}L D L R \\
A P O B\end{array}$ & $\begin{array}{l}66 \text { LDLR } \\
\text { mutations } \\
10 A P O B \\
\text { mutations }\end{array}$ \\
\hline & $\begin{array}{c}\text { Japan } \\
{[34]}\end{array}$ & $\begin{array}{l}\text { criteria suggested } \\
\text { by the Japan } \\
\text { Atherosclerosis } \\
\text { Society }\end{array}$ & 205 & 118 & $\begin{array}{l}\text { SSCP assay } \\
\text { MLPA }\end{array}$ & $L D L R$ & $\begin{array}{l}53 \text { LDLR } \\
\text { mutations } \\
21 \text { large } \\
\text { rearrange- } \\
\text { ments }\end{array}$ \\
\hline
\end{tabular}

Legend: $L D L R$-low-density lipoprotein receptor, $A P O B$ - apolipoprotein B; PCSK9—proprotein convertase subtilisin/kexin type 9, DLCN-Dutch Lipid Clinic Network, HeFH—heterozygous form of familial hypercholesterolemia, SB- Simon Broome, MLPA-multiplex ligation-dependent probe amplification, RT PCR-reverse transcription-polymerase chain reaction, NGS-next-generation sequencing, SSCP_single-strand conformation polymorphism, ARMS-PCR—tetra-primer amplification refractory mutation system-polymerase chain reaction, PCR-RFLP—restriction fragment length polymorphism, DDGE—denaturing gradient gel electrophoresis. 
In our study, we recognized one patient with a pathogenic mutation-duplication of part of the LDLR, exhibited by the exon 13-15 duplication (c.(1845+1_1846-1)_(2311+1_23121)dup)). In one Italian FH patient, based on Southern blot analysis, Lelli et al. observed the insertion caused by a duplication of exons 13,14, and 15, being the result of an unequal crossover between repetitive sequences located in intron 12 and intron 15, which was titled FH Bologna-2 [48]. Likewise, this duplication was identified in one patient in the study conducted by Futema et al., which included 48 patients with definite FH from the UK [36].

At the same time, the FH phenotype can be explained by mutations in the $A P O B$ and PCSK9 genes [30], which have not been detected so far in Romania. As described above, a higher prevalence of $L D L R$ variants and a lower number of variants in $A P O B$ and $P C S K 9$ were observed [49]. In our study, $A P O B$ and PCSK9 mutations were synonymous, benign, and patients with these variants probably did not have a monogenic disorder of lipid metabolism, but they had a polygenic form of hypercholesterolemia. Madeira et al. reached the same conclusions in the study conducted on FH patients in Portugal [49].

Depending on the DLCN score, the subjects with possible FH had $9.8 \%$ mutations, those with probable $\mathrm{FH}$ had $21.3 \%$ mutations, and individuals with definite $\mathrm{FH}$ had $6.5 \%$ mutations, while in an FH cohort from Italy, Bertolini et al. showed that the mutation detection rate of subjects stratified according to the DLCN score was "definite $\mathrm{FH}^{\text {" - }}$ $91.9 \%$, "probable $\mathrm{FH}^{\prime}-76.6 \%$, and "possible $\mathrm{FH}^{\prime}-69.4 \%$ [2]. The definite $\mathrm{FH}$ patients had pathogenic mutations in $4.9 \%$ of cases, whereas in $8.2 \%$ of cases, no mutations were identified. The polygenic component may provide a possible explanation in this respect and future studies acknowledging these observations would be worth conducting.

Although all of the identified homozygous and heterozygous patients with pathogenic/likely pathogenic mutations were treated with lipid-lowering drugs, none of them achieved the recommended LDL-C targets $(<55 \mathrm{mg} / \mathrm{dL})$ of guidelines on dyslipidemia [24]; different therapeutic approaches are necessary in order to decrease the high cardiovascular risk of these patients [24,49].

In 38 patients included in this study, no mutations of $L D L R$, PCSK9, and APOB were detected by MLPA analysis, by exon-by-exon sequencing of amplified genomic DNA. Among patients without mutations, $8.2 \%$ had a definite $\mathrm{FH}$ clinical diagnosis, involving an inherited component of their disorder, which may not be monogenic or may result from more complex interactions between gene variants and the environment [35]. However, despite the addition of LDLRAP1 and PCSK9 to the list of genes that are associated with inherited hypercholesterolemia, there still appears to be a substantial number of patients with a clinical diagnosis of possible or defined FH who exhibit unknown genetic defect [35]. In a study that enrolled 61.217 patients, only 5.4\% were diagnosed with inherited atherogenic dyslipidemia, reported primarily as a secondary diagnosis; this pathology was identified in a much smaller number of cases compared to the real number of patients, mainly because of the short period of hospitalization for acute coronary syndromes [50].

For studying genotype-phenotype interactions in a genetically homogeneous population Romanian, the analysis of the effect of mutation on the variation of plasma cholesterol levels and the expression of cardiovascular events were important, a concept also supported by Weiss et al. [37]. The pathogenic/likely pathogenic $L D L R$ mutations were significant predictors of the new cardiovascular events, and the time interval for new cardiovascular events occurrence was significantly decreased, compared to $\mathrm{FH}$ patients without mutations. In our study, the patients with pathogenic/likely pathogenic mutations, even if they had a reduced chronic inflammation status (hsCRP with low values), expressed significantly expanded levels upon lipid profile under lipid-lowering treatment throughout the follow up, compared to patients without mutations with ASCVD. Moreover, at the end of the study, patients with pathogenic mutations recorded significantly increased cIMT and decreased $\mathrm{EF}$, compared to those with ASCVD but without mutations.

$\mathrm{Lp}(\mathrm{a})$ levels are associated with a high risk of cardiovascular mortality, requiring a new pharmacotherapeutic approach, and 5-20\% of patients suspected of $\mathrm{FH}$ had elevated 
Lp(a) levels [51]. In our study, we did not measure the Lp(a) values, but these findings will be exploited in a future study in which the $\mathrm{Lp}(\mathrm{a})$ of the patients will be assessed.

The statin treatment has been frequently used in our study and triple therapy has been frequently indicated for patients with pathogenic mutations. The beneficial effect of statins on cardiovascular events is due to their cholesterol-lowering properties, as inhibition of 3-hydroxy-3-methyl-glutaryl-coenzyme A (HMG-CoA) reductase can lead to pleiotropic effects. Statins have an important anti-inflammatory effect by decreasing the number of LDL particles found in the vascular wall. In addition, ezetimibe reduces the absorption of intestinal cholesterol, and along with statins, contribute to further lowering of LDLC and cardiovascular events [13].

\section{Study Strengths and Limitations}

This is the first observational genetic approach in Romania that included patients with FH in order to identify the relationship between specific mutations in LDLR, APOB, PCSK9, and ASCVD. This study represents an important step in identifying cases with FH and ASCVD to create a bridge between specialties (cardiology—internal medicine-genetics). Genetic testing is essential to confirm the diagnosis of FH based on clinical and paraclinical components, despite the fact that it is not currently performed in Romania or reimbursed by the Romanian health system [52]. In the absence of any specific screening program, the percentage of undiagnosed FH carriers may be increased, leading to significant cardiovascular events. However, in practice, the diagnosis is based on the calculation of clinical risk scores related to DLCN, Simon Broome, and MedPed criteria. The clinical identification, complemented by genetic recognition of $\mathrm{FH}$ patients contributes to the improvement of the management and to the ASCVD decline [52]. Furthermore, these data must benefit from a special platform patient data and the results of the molecular genetics analyses should be introduced [52]. Another strength is that the same mutation on exon 11 of the LDLR gene (c.1618G > A) with homozygous status was identified in several patients with FH.

Nonetheless, this study had some limitations. Firstly, the methodology of the study was observational. Secondly, a small number of patients were included in the study (because for most eligible patients there were no values for EF, ABI, or lipid profile prior to their inclusion in the study). Thirdly, the current study enrolled subjects from the northeastern area of Romania, and thus, the current group of patients did not significantly reflect the entire Romanian population with ASCVD. No cascade studies were performed to examine segregation and genotype-phenotype interactions regarding the three mutations. A possible cause of the decreased frequency of LDLR mutations in our FH patients could be due to the fact that the genetic defects were benign or likely benign.

\section{Conclusions}

In this study, out of $61 \mathrm{FH}$ patients included based on the DCLN score over 3, seven patients had pathogenic/likely pathogenic variants and 16 benign/likely benign variants, confirmed by MLPA and Sanger sequencing. Four pathogenic variants have been recognized, i.e., c.81C > G (exon 2 of $L D L R$ gene) with heterozygous form, c.502G > A (exon 4 of LDLR gene) with heterozygous form, c.1618G > A (exon 11 of LDLR gene) with homozygous form, and exon 13-15 duplication (c.(1845+1_1846-1)_(2311+1_2312-1)dup)) in $L D L R$ genes, which have not been reported in any study conducted in Romania. In patients with pathogenic/likely pathogenic mutations, the hsCRP and HDL-C levels were decreased, while TC and LDL-C levels were increased under lipid-lowering treatment throughout the follow up. Additionally, the pathogenic/likely pathogenic LDLR mutations were significant predictors of the new cardiovascular events, and the time interval for the occurrence of new cardiovascular events was significantly decreased, compared to $\mathrm{FH}$ patients without mutations.

Supplementary Materials: The following are available online at https: / www.mdpi.com/article/10 $.3390 / \mathrm{jcm} 10071399 / \mathrm{s} 1$, Table S1: LDLR, APOB, and PCSK9 variants identified in the Romanian FH cohort and not reported previously in this population. 
Author Contributions: Conceptualization, C.-E.V., L.G.F., and V.T.; data curation, R.A. and D.R.-P.; formal analysis, L.G.F., I.P., and V.T.; investigation, C.-E.V., R.P., I.P., and D.R.-P.; methodology, R.P., D.R.-P., and A.C.; supervision, M.K. and A.C.; validation, R.P., D.R.-P., L.F., and A.C.; writingoriginal draft preparation, C.-E.V.; writing-review and editing, L.G.F., M.K., and A.C. All authors have read and agreed to the published version of the manuscript.

Funding: This research received no external funding.

Institutional Review Board Statement: The study was conducted according to the guidelines of the Declaration of Helsinki and approved by the Ethics Committee of "Dr. C. I. Parhon" Clinical Hospital (09.2017), Ethics Committee of "Grigore T. Popa" the University of Medicine and Pharmacy Iasi (approval No. 5071/5.03.2018), and the Ethics Commission of "Prof. dr. George IM Georgescu" Institute of Cardiovascular Diseases, Iasi (approval No. 2285/20.06.2018), Ethics Commission of "Sf. Spiridon" Emergency Clinical Hospital (approval No. 22/1.08.2018).

Informed Consent Statement: Informed consent was obtained from all subjects involved in the study.

Data Availability Statement: Not applicable.

Conflicts of Interest: The authors declare no conflict of interest.

\section{References}

1. Trinder, M.; Li, X.; DeCastro, M.L.; Cermakova, L.; Sadananda, S.; Jackson, L.M.; Azizi, H.; Mancini, G.B.J.; Francis, G.A.; Frohlich, J.; et al. Risk of Premature Atherosclerotic Disease in Patients With Monogenic Versus Polygenic Familial Hypercholesterolemia. J. Am. Coll. Cardiol. 2019, 74, 512-522. [CrossRef]

2. Bertolini, S.; Pisciotta, L.; Rabacchi, C.; Cefalu, A.B.; Noto, D.; Fasano, T.; Signori, A.; Fresa, R.; Averna, M.; Calandra, S. Spectrum of mutations and phenotypic expression in patients with autosomal dominant hypercholesterolemia identified in Italy. Atherosclerosis 2013, 227, 342-348. [CrossRef] [PubMed]

3. Gabcova, D.; Vohnout, B.; Stanikova, D.; Huckova, M.; Kadurova, M.; Debreova, M.; Kozarova, M.; Fabryova, L.; Stanik, J.; Klimes, I.; et al. The molecular genetic background of familial hypercholesterolemia: Data from the Slovak nation-wide survey. Physiol. Res. 2017, 66, 75-84. [CrossRef] [PubMed]

4. Jannes, C.E.; Santos, R.D.; de Souza Silva, P.R.; Turolla, L.; Gagliardi, A.C.; Marsiglia, J.D.; Chacra, A.P.; Miname, M.H.; Rocha, V.Z.; Filho, W.S.; et al. Familial hypercholesterolemia in Brazil: Cascade screening program, clinical and genetic aspects. Atherosclerosis 2015, 238, 101-107. [CrossRef] [PubMed]

5. Cymbron, T.; Mendes, P.; Ramos, A.; Raposo, M.; Kazachkova, N.; Medeiros, A.M.; Bruges-Armas, J.; Bourbon, M.; Lima, M. Familial hypercholesterolemia: Molecular characterization of possible cases from the Azores Islands (Portugal). Meta Gene 2014, 2, 638-645. [CrossRef]

6. Benito-Vicente, A.; Uribe, K.B.; Jebari, S.; Galicia-Garcia, U.; Ostolaza, H.; Martin, C. Validation of LDLr Activity as a Tool to Improve Genetic Diagnosis of Familial Hypercholesterolemia: A Retrospective on Functional Characterization of LDLr Variants. Int. J. Mol. Sci. 2018, 19, 1676. [CrossRef] [PubMed]

7. Paththinige, C.S.; Rajapakse, J.; Constantine, G.R.; Sem, K.P.; Singaraja, R.R.; Jayasekara, R.W.; Dissanayake, V.H.W. Spectrum of low-density lipoprotein receptor (LDLR) mutations in a cohort of Sri Lankan patients with familial hypercholesterolemia-A preliminary report. Lipids Health Dis. 2018, 17, 100. [CrossRef]

8. Ekrami, M.; Torabi, M.; Ghafouri-Fard, S.; Mowla, J.; Mohammad Soltani, B.; Hashemi-Gorji, F.; Mohebbi, Z.; Miryounesi, M. Genetic Analysis of Iranian Patients with Familial Hypercholesterolemia. Iran. Biomed. J. 2018, 22, 117-122.

9. Sharifi, M.; Walus-Miarka, M.; Idzior-Walus, B.; Malecki, M.T.; Sanak, M.; Whittall, R.; Li, K.W.; Futema, M.; Humphries, S.E. The genetic spectrum of familial hypercholesterolemia in south-eastern Poland. Metabolism 2016, 65, 48-53. [CrossRef]

10. Al-Khateeb, A.; Zahri, M.K.; Mohamed, M.S.; Sasongko, T.H.; Ibrahim, S.; Yusof, Z.; Zilfalil, B.A. Analysis of sequence variations in low-density lipoprotein receptor gene among Malaysian patients with familial hypercholesterolemia. BMC Med. Genet. 2011, 12, 40. [CrossRef]

11. Faiz, F.; Allcock, R.J.; Hooper, A.J.; van Bockxmeer, F.M. Detection of variations and identifying genomic breakpoints for large deletions in the LDLR by Ion Torrent semiconductor sequencing. Atherosclerosis 2013, 230, 249-255. [CrossRef]

12. Rahman, T.; Hamzan, N.S.; Mokhsin, A.; Rahmat, R.; Ibrahim, Z.O.; Razali, R.; Thevarajah, M.; Nawawi, H. Enhanced status of inflammation and endothelial activation in subjects with familial hypercholesterolaemia and their related unaffected family members: A case control study. Lipids Health Dis. 2017, 16, 81. [CrossRef]

13. Ruscica, M.; Corsini, A.; Ferri, N.; Banach, M.; Sirtori, C.R. Clinical approach to the inflammatory etiology of cardiovascular diseases. Pharmacol. Res. 2020, 159, 104916. [CrossRef]

14. Jeenduang, N.; Promptmas, C.; Pongrapeeporn, K.S.; Porntadavity, S. Molecular modeling of D151Y and M391T mutations in the LDL receptor. Biochem. Biophys. Res. Commun. 2008, 377, 355-360. [CrossRef] 
15. Maurer, F.; Pradervand, S.; Guilleret, I.; Nanchen, D.; Maghraoui, A.; Chapatte, L.; Bojkowska, K.; Bhuiyan, Z.A.; Jacquemont, N.; Harshman, K.; et al. Identification and molecular characterisation of Lausanne Institutional Biobank participants with familial hypercholesterolaemia-A proof-of-concept study. Swiss Med. Wkly. 2016, 146, w14326. [CrossRef]

16. Mozas, P.; Castillo, S.; Tejedor, D.; Reyes, G.; Alonso, R.; Franco, M.; Saenz, P.; Fuentes, F.; Almagro, F.; Mata, P.; et al. Molecular characterization of familial hypercholesterolemia in Spain: Identification of 39 novel and 77 recurrent mutations in LDLR. Hum. Mutat. 2004, 24, 187. [CrossRef]

17. Blanchard, V.; Khantalin, I.; Ramin-Mangata, S.; Chemello, K.; Nativel, B.; Lambert, G. PCSK9: From biology to clinical applications. Pathology 2019, 51, 177-183. [CrossRef] [PubMed]

18. Seidah, N.G.; Benjannet, S.; Wickham, L.; Marcinkiewicz, J.; Jasmin, S.B.; Stifani, S.; Basak, A.; Prat, A.; Chretien, M. The secretory proprotein convertase neural apoptosis-regulated convertase 1 (NARC-1): Liver regeneration and neuronal differentiation. Proc. Natl. Acad. Sci. USA 2003, 100, 928-933. [CrossRef] [PubMed]

19. Macchi, C.; Banach, M.; Corsini, A.; Sirtori, C.R.; Ferri, N.; Ruscica, M. Changes in circulating pro-protein convertase subtilisin/kexin type 9 levels-Experimental and clinical approaches with lipid-lowering agents. Eur. J. Prev. Cardiol. 2019, 26, 930-949. [CrossRef] [PubMed]

20. Vlad, C.E.; Foia, L.; Popescu, R.; Ivanov, I.; Luca, M.C.; Delianu, C.; Toma, V.; Statescu, C.; Rezus, C.; Florea, L. Apolipoproteins A and B and PCSK9: Nontraditional Cardiovascular Risk Factors in Chronic Kidney Disease and in End-Stage Renal Disease. J. Diabetes Res. 2019, 2019, 6906278. [CrossRef] [PubMed]

21. Rogacev, K.S.; Heine, G.H.; Silbernagel, G.; Kleber, M.E.; Seiler, S.; Emrich, I.; Lennartz, S.; Werner, C.; Zawada, A.M.; Fliser, D.; et al. PCSK9 Plasma Concentrations Are Independent of GFR and Do Not Predict Cardiovascular Events in Patients with Decreased GFR. PLoS ONE 2016, 11, e0146920. [CrossRef] [PubMed]

22. Defesche, J.C.; Gidding, S.S.; Harada-Shiba, M.; Hegele, R.A.; Santos, R.D.; Wierzbicki, A.S. Familial hypercholesterolaemia. Nat. Rev. Dis. Primers 2017, 3, 17093. [CrossRef] [PubMed]

23. Mach, F.; Baigent, C.; Catapano, A.L.; Koskinas, K.C.; Casula, M.; Badimon, L.; Chapman, M.J.; De Backer, G.G.; Delgado, V.; Ference, B.A.; et al. 2019 ESC/EAS Guidelines for the management of dyslipidaemias: Lipid modification to reduce cardiovascular risk: The Task Force for the management of dyslipidaemias of the European Society of Cardiology (ESC) and European Atherosclerosis Society (EAS). Eur. Heart J. 2020, 41, 111-188. [CrossRef] [PubMed]

24. Mach, F.; Baigent, C.; Catapano, A.L.; Koskinas, K.C.; Casula, M.; Badimon, L.; Chapman, M.J.; De Backer, G.G.; Delgado, V.; Ference, B.A.; et al. 2019 ESC/EAS guidelines for the management of dyslipidaemias: Lipid modification to reduce cardiovascular risk. Atherosclerosis 2019, 290, 140-205. [CrossRef]

25. Perez de Isla, L.; Alonso, R.; Mata, N.; Fernandez-Perez, C.; Muniz, O.; Diaz-Diaz, J.L.; Saltijeral, A.; Fuentes-Jimenez, F.; de Andres, R.; Zambon, D.; et al. Predicting Cardiovascular Events in Familial Hypercholesterolemia: The SAFEHEART Registry (Spanish Familial Hypercholesterolemia Cohort Study). Circulation 2017, 135, 2133-2144. [CrossRef] [PubMed]

26. Khan, S.P.; Ahmed, K.Z.; Yaqub, Z.; Ghani, R. Carotid intima-media thickness correlation with lipid profile in patients with familial hypercholesterolemia versus controls. J. Coll. Physicians Surg. Pak. 2011, 21, 30-33. [PubMed]

27. Sivapalaratnam, S.; van Loendersloot, L.L.; Hutten, B.A.; Kastelein, J.J.; Trip, M.D.; de Groot, E. Long-term LDL-c lowering in heterozygous familial hypercholesterolemia normalizes carotid intima-media thickness. Atherosclerosis 2010, 212, 571-574. [CrossRef] [PubMed]

28. Resmerita, I.; Cozma, R.S.; Popescu, R.; Radulescu, L.M.; Panzaru, M.C.; Butnariu, L.I.; Caba, L.; Ilie, O.D.; Gavril, E.C.; Gorduza, E.V.; et al. Genetics of Hearing Impairment in North-Eastern Romania-A Cost-Effective Improved Diagnosis and Literature Review. Genes 2020, 11, 1506. [CrossRef]

29. Schwarz, J.M.; Rodelsperger, C.; Schuelke, M.; Seelow, D. MutationTaster evaluates disease-causing potential of sequence alterations. Nat. Methods 2010, 7, 575-576. [CrossRef]

30. Mollaki, V.; Progias, P.; Drogari, E. Familial Hypercholesterolemia in Greek children and their families: Genotype-to-phenotype correlations and a reconsideration of LDLR mutation spectrum. Atherosclerosis 2014, 237, 798-804. [CrossRef]

31. Iacocca, M.A.; Chora, J.R.; Carrie, A.; Freiberger, T.; Leigh, S.E.; Defesche, J.C.; Kurtz, C.L.; DiStefano, M.T.; Santos, R.D.; Humphries, S.E.; et al. ClinVar database of global familial hypercholesterolemia-associated DNA variants. Hum. Mutat. 2018, 39, 1631-1640. [CrossRef] [PubMed]

32. National Center for Biotechnology Information ClinVar. 2020. Available online: https://www.ncbi.nlm.nih.gov/clinvar/ (accessed on 19 February 2021).

33. Richards, S.; Aziz, N.; Bale, S.; Bick, D.; Das, S.; Gastier-Foster, J.; Grody, W.W.; Hegde, M.; Lyon, E.; Spector, E.; et al. Standards and guidelines for the interpretation of sequence variants: A joint consensus recommendation of the American College of Medical Genetics and Genomics and the Association for Molecular Pathology. Genet. Med. 2015, 17, 405-424. [CrossRef] [PubMed]

34. Miyake, Y.; Yamamura, T.; Sakai, N.; Miyata, T.; Kokubo, Y.; Yamamoto, A. Update of Japanese common LDLR gene mutations and their phenotypes: Mild type mutation L547V might predominate in the Japanese population. Atherosclerosis 2009, 203, 153-160. [CrossRef] [PubMed]

35. Tosi, I.; Toledo-Leiva, P.; Neuwirth, C.; Naoumova, R.P.; Soutar, A.K. Genetic defects causing familial hypercholesterolaemia: Identification of deletions and duplications in the LDL-receptor gene and summary of all mutations found in patients attending the Hammersmith Hospital Lipid Clinic. Atherosclerosis 2007, 194, 102-111. [CrossRef] 
36. Futema, M.; Plagnol, V.; Whittall, R.A.; Neil, H.A.; Simon Broome Register, G.; Humphries, S.E. Uk10K Use of targeted exome sequencing as a diagnostic tool for Familial Hypercholesterolaemia. J. Med. Genet. 2012, 49, 644-649. [CrossRef] [PubMed]

37. Weiss, N.; Binder, G.; Keller, C. Mutations in the low-density-lipoprotein receptor gene in German patients with familial hypercholesterolaemia. J. Inherit. Metab. Dis. 2000, 23, 778-790. [CrossRef]

38. Dedoussis, G.V.; Skoumas, J.; Pitsavos, C.; Choumerianou, D.M.; Genschel, J.; Schmidt, H.; Stefanadis, C. FH clinical phenotype in Greek patients with LDL-R defective vs. negative mutations. Eur. J. Clin. Investig. 2004, 34, 402-409. [CrossRef]

39. Komarova, T.Y.; Korneva, V.A.; Kuznetsova, T.Y.; Golovina, A.S.; Vasilyev, V.B.; Mandelshtam, M.Y. Familial hypercholesterolemia mutations in Petrozavodsk: No similarity to St. Petersburg mutation spectrum. BMC Med. Genet. 2013, 14, 128. [CrossRef]

40. Zakharova, F.M.; Damgaard, D.; Mandelshtam, M.Y.; Golubkov, V.I.; Nissen, P.H.; Nilsen, G.G.; Stenderup, A.; Lipovetsky, B.M.; Konstantinov, V.O.; Denisenko, A.D.; et al. Familial hypercholesterolemia in St-Petersburg: The known and novel mutations found in the low density lipoprotein receptor gene in Russia. BMC Med. Genet. 2005, 6, 6. [CrossRef]

41. Gorski, B.; Kubalska, J.; Naruszewicz, M.; Lubinski, J. LDL-R and Apo-B-100 gene mutations in Polish familial hypercholesterolemias. Hum. Genet. 1998, 102, 562-565. [CrossRef]

42. Lopez, G.; Bernal, L.M.; Gelvez, N.; Gomez, L.F.; Nova, A.; Sanchez, A.I.; Tamayo, M.L. Mutational analysis of the LDLR gene in a cohort of Colombian families with familial hypercholesterolemia. Atherosclerosis 2018, 277, 434-439. [CrossRef]

43. Al-Allaf, F.A.; Athar, M.; Abduljaleel, Z.; Bouazzaoui, A.; Taher, M.M.; Own, R.; Al-Allaf, A.F.; AbuMansour, I.; Azhar, Z.; Ba-Hammam, F.A.; et al. Identification of a novel nonsense variant c.1332dup, p.(D445*) in the LDLR gene that causes familial hypercholesterolemia. Hum. Genome Var. 2014, 1, 14021. [CrossRef]

44. Chiou, K.R.; Charng, M.J.; Chang, H.M. Array-based resequencing for mutations causing familial hypercholesterolemia. Atherosclerosis 2011, 216, 383-389. [CrossRef]

45. Day, I.N.; Whittall, R.A.; O’Dell, S.D.; Haddad, L.; Bolla, M.K.; Gudnason, V.; Humphries, S.E. Spectrum of LDL receptor gene mutations in heterozygous familial hypercholesterolemia. Hum. Mutat. 1997, 10, 116-127. [CrossRef]

46. Etxebarria, A.; Benito-Vicente, A.; Stef, M.; Ostolaza, H.; Palacios, L.; Martin, C. Activity-associated effect of LDL receptor missense variants located in the cysteine-rich repeats. Atherosclerosis 2015, 238, 304-312. [CrossRef] [PubMed]

47. Tichy, L.; Fajkusova, L.; Zapletalova, P.; Schwarzova, L.; Vrablik, M.; Freiberger, T. Molecular genetic background of an autosomal dominant hypercholesterolemia in the Czech Republic. Physiol. Res. 2017, 66, S47-S54. [CrossRef] [PubMed]

48. Lelli, N.; Ghisellini, M.; Calandra, S.; Gaddi, A.; Ciarrocchi, A.; Coviello, D.A.; Bertolini, S. Duplication of exons 13, 14 and 15 of the LDL-receptor gene in a patient with heterozygous familial hypercholesterolemia. Hum. Genet. 1991, 86, 359-362. [CrossRef]

49. Medeiros, A.M.; Alves, A.C.; Bourbon, M. Mutational analysis of a cohort with clinical diagnosis of familial hypercholesterolemia: Considerations for genetic diagnosis improvement. Genet. Med. 2016, 18, 316-324. [CrossRef]

50. Sampietro, T.; Pieroni, S.; Molinaro, S.; Sbrana, F.; Dal Pino, B.; Bigazzi, F.; Ruscica, M.; Sirtori, C.R.; Franchini, M. Inherited atherogenic dyslipidemias: Are they correctly reported? Eur. J. Prev. Cardiol. 2020, 12, 2047487320930308. [CrossRef]

51. Greco, M.F.; Sirtori, C.R.; Corsini, A.; Ezhov, M.; Sampietro, T.; Ruscica, M. Lipoprotein(a) Lowering-From Lipoprotein Apheresis to Antisense Oligonucleotide Approach. J. Clin. Med. 2020, 9, 2103. [CrossRef]

52. Vlad, C.E.; Foia, L.; Florea, L.; Costache, I.I.; Covic, A.; Popescu, R.; Reurean-Pintilei, D.; Covic, A. Evaluation of cardiovascular risk factors in patients with familial hypercholesterolemia from the North-Eastern area of Romania. Lipids Health Dis. 2021, 20, 4. [CrossRef] [PubMed] 